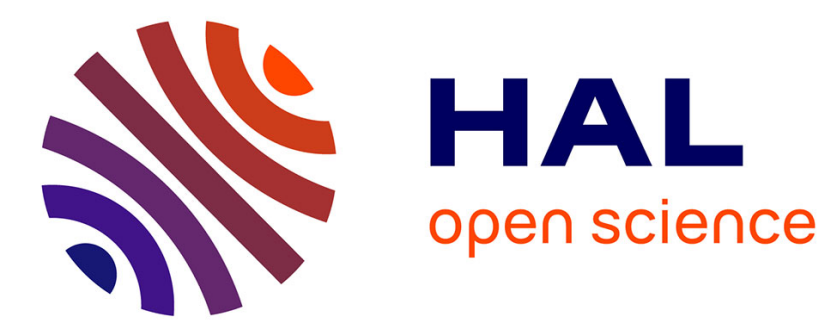

\title{
Influence of wall vibrations on the behavior of a simplified wind instrument
}

Guillaume Nief, François Gautier, Jean-Pierre Dalmont, Joël Gilbert

\section{To cite this version:}

Guillaume Nief, François Gautier, Jean-Pierre Dalmont, Joël Gilbert. Influence of wall vibrations on the behavior of a simplified wind instrument. Journal of the Acoustical Society of America, 2008, 124 (2), pp.1320 - 1331. hal-00331386

\section{HAL Id: hal-00331386 https://hal.science/hal-00331386}

Submitted on 16 Oct 2008

HAL is a multi-disciplinary open access archive for the deposit and dissemination of scientific research documents, whether they are published or not. The documents may come from teaching and research institutions in France or abroad, or from public or private research centers.
L'archive ouverte pluridisciplinaire HAL, est destinée au dépôt et à la diffusion de documents scientifiques de niveau recherche, publiés ou non, émanant des établissements d'enseignement et de recherche français ou étrangers, des laboratoires publics ou privés. 
Influence of wall vibrations on the behavior of a simplified wind instrument

Guillaume Nief,* François Gautier, Jean-Pierre Dalmont, and Joël Gilbert

Laboratoire d'Acoustique de l'Université du Maine, UMR CNRS 6613, Av. O. Messiaen, 72085 Le Mans Cedex 9, France.

(Dated: May 19, 2008) 


\begin{abstract}
The issue of the influence of wall vibrations on the behavior of wind instruments is still debated. The mechanisms of vibroacoustic couplings involved by these vibrations are difficult to investigate, as fluidstructure interactions are weak. Among these vibroacoustic interactions, the present study is focused on the coupling between the internal acoustic field and the mechanical behavior of the duct. For this purpose, a simplified single reed instrument consisting of a brass tube connected to a clarinet mouthpiece has been studied. A theoretical model of coupling between the plane inner acoustic wave and mechanical modes is developed and suggests that in order to obtain measurable effects of wall vibrations, the geometrical parameters of the studied tube have to be unusual compared to that of real instruments. For a slightly oval-shaped and very thin brass tube, it is shown theoretically and experimentally that a coupling between the inner plane acoustic wave and ovalling mechanical modes occurs, and results in disturbances of the input impedance, which can slightly affect the tone color of the sound produced. It is concluded that the reported effects are unlikely to occur in real instruments except for some organ pipes.
\end{abstract}

PACS numbers: 43.75.Pq 


\section{INTRODUCTION}

In the past few decades, much progress has been made toward a better understanding of wind instruments ${ }^{1-3}$. Their main physical principles of operation are nowadays quite well understood. Nevertheless, a few topics are still debated. The issue of the effects of wall vibrations on the behavior of wind instruments is one of them. Walls are usually considered as infinitely stiff compared to the enclosed air and thus perfectly rigid. However, wall vibrations can easily be experienced by any player since they can be felt during playing. Optical holography techniques have also been used to observe such vibrations ${ }^{4}$. Moreover, they are claimed to be of great influence by many musicians and instrument makers. As a consequence, this subject which is often associated with the influence of the constitutive material of the instrument, has been studied since quite early days in the history of musical acoustics of wind instruments ${ }^{5,6}$. However, the past studies dealing with this topic are not very numerous and have produced mixed results. These studies can be classified into groups considering the origin of the vibrations and their effects.

The various vibroacoustic couplings involved in a musical instrument have been investigated theoretically ${ }^{7}$. Two different sources may generate wall vibrations, an acoustical one and a mechanical one. Firstly, the acoustical source involves the excitation of the walls by the inner pressure field. Secondly, the impacts of the reed on the mouthpiece for single reed instruments or direct transmission from vibrations of the lips for brass instruments are mechanical sources which can be responsible for wall vibrations.

Whitehouse $^{9}$ investigated the source of the wall vibrations on a simplified brass instrument: a trombone mouthpiece connected to a simple tube. By coupling or uncoupling the lips to the tube or the air column to the tube, he concluded that the mechanical source of vibration was stronger than the acoustical one. For the excitation of the walls by the inner pressure field, Watkinson and Bowsher ${ }^{8}$ studied the in vacuo modes of trombone bells with varying material and geometrical properties using a finite element technique. They calculated the

*Electronic address: guillaume.nief .etu@univ-lemans.fr 
mechanical response to the internal acoustic field. They found that the modes usually came grouped by pairs with slightly different associated eigenfrequencies and normal modal shapes due to the asymmetry of the system. They also observed that the inner acoustic plane wave couples only with non "perfectly symmetrical" mode shapes.

Although the fluid-structure interactions in wind instruments are weak, the vibrations of the walls, no matter their origin, may then induce sound radiation in the surrounding external or internal fluid. Direct radiation to the external fluid may contribute to the overall radiated field. The radiation to the internal fluid may induce a perturbation of the oscillations of the air column and thus disturb indirectly the radiated field. For the coupling with the inner acoustic field, Yousri and Fahy ${ }^{11}$ described theoretically, with a non musical aim, the coupling between the modes of a cylindrical shell and the inner plane acoustical wave below the cut off frequency of the first helical mode. The non axisymmetrical mechanical modes can be coupled only if the cylindrical shell is geometrically slightly distorted, or presents inhomogeneous material properties. Backus ${ }^{10}$ investigated the effects of the vibration of walls considering both the external radiation from the instrument's body and the possible air column alteration. He concluded that the vibrations are extremely weak and do not affect the steady tones for both of the considered coupling mechanisms. On the contrary, Nederveen and Dalmont ${ }^{12}$ have observed a spectacular effect of wall vibrations in the case of an organ pipe, the bifurcation towards a pseudo periodic regime. Nief et al. ${ }^{27}$ have reported similar effects in the case of a very thin plastic tube connected to a clarinet mouthpiece. Moreover, Pico and Gautier ${ }^{17}$ have developed a multimodal coupling model and have shown theoretically that the input impedance of acoustic waves in the pipe could be slightly disturbed by wall vibrations.

Among the possible couplings, the present article studies the coupling between the inner fluid and wall vibrations. It is supposed that the walls are excited by the inner pressure field, and it is investigated how the resulting vibrations can have an influence on the input impedance and on the produced sounds. This study is carried out on a simplified instrument composed of a single reed and a simple straight tube. In section 2 , theoretical considera- 
tions are given for the input impedance of a vibrating tube and for the instrument in playing condition, and show that to obtain a measurable effect, the tested tube has to be very thin $(0.2 \mathrm{~mm})$ and slightly oval-shaped, which is not realistic for a wind instrument. In section 3, experimental mechanical modal analysis and acoustical input impedance measurements are presented in order to study in detail the vibroacoustic coupling. The system is also tested in playing condition to investigate the effects on sound produced in terms of spectral content and playing frequency. The measured effects are then interpreted using the physical model in playing situation which allows the musical sounds to be simulated.

\section{THEORETICAL CONSIDERATIONS}

\section{A. Acoustic input impedance of a tube and influence of wall vibrations}

\section{Input impedance of a rigid tube}

A musical wind instrument can be described as a coupled exciter-resonator system. The exciter can be characterised by a non-linear relation between acoustical pressure and acoustical volume velocity ${ }^{13}$. The resonator can be characterised by a simple linear proportionality in the frequency domain between the acoustical pressure $P(\omega)$ and the acoustic volume velocity $U(\omega)$, where $\omega$ is the angular frequency (Eq.1).

$$
Z(\omega)=\frac{P(\omega)}{U(\omega)}
$$

Non linear phenomena such as shock waves, or non linear losses at open ends have sometimes to be taken into consideration when describing the acoustic resonator, but they are not considered here. The quantity $Z(\omega)$ expressed at the input of the resonator is the acoustic input impedance. It depends mainly on the bore shape of the tube and is often used for the study of the resonator part of a wind instrument. Indeed, it can be linked to important physical properties of the instrument under musical performance. The positions of the peaks are strongly linked with the playing frequency and thus with the intonation of the instrument ${ }^{14}$. Input impedance characteristics are also related to timbre and facility of sound 
production of different notes ${ }^{15}$.

For a cylindrical and perfectly rigid tube open at the end, considering only the plane acoustic wave and taking into account the length correction due to radiation, the acoustic input impedance can be written as :

$$
Z_{r}(\omega)=\frac{\rho_{0} c_{0}}{S} j \tan (k(\omega) L)
$$

where $c_{0}$ is the speed of sound, $\rho_{0}$ the air density, $L$ the equivalent length of the tube taking into account the end correction due to radiation and $S$ its cross-sectional area. $k(\omega)$ is the complex wave number taking into account the viscous and thermal losses at the walls ${ }^{16}$ :

$$
k(\omega)=k_{0}\left(1+\frac{\sqrt{2}(1-j)}{a \sqrt{k_{0}}}\left(\sqrt{l_{v}}+(\gamma-1) \sqrt{l_{h}}\right)\right)^{1 / 2}
$$

where $k_{0}=\omega / c_{0}, l_{v} \approx 410^{-8} \mathrm{~m}$ is the characteristic length for viscous effects in air, $l_{h} \approx$ $5.610^{-8} \mathrm{~m}$ is the characteristic length for the thermal effects in air and $\gamma=1.4$ is the ratio of specific heats for air. The index $r$ associated with the input impedance indicates that Eq.2 is the expression for a rigid tube. The modulus of this input impedance $Z_{r}$ shows peaks corresponding to the acoustical resonances of the closed-open air column.

\section{Correction factor to input impedance due to the wall vibration}

The previous description does not take into account possible wall vibrations. In order to describe those vibrations, it is necessary to consider that the tube behaves as an elastic shell. The instrument is modelled in a simple way by a cylindrical shell clamped on one end and free on the other. The shell is excited by the internal acoustic pressure field and set into vibration. The vibrations induce a disturbance of the initial pressure field. This modification of the air column oscillation yields a slight modification of the input impedance of the perfectly rigid tube ${ }^{17}$. In this approach, structural vibrations and inner acoustic pressure are coupled. The central point for modelling the acoustic input impedance of the vibrating tube is to take into account a slight ellipticity of the tube, which models the effective asymmetry. In a first approximation, its radius $r$ can be written using the polar equation which is plotted 
on FIG.1(a):

$$
r(\theta)=a(1+\epsilon \cos (2 \theta))
$$

where $a$ denotes the mean radius and $\epsilon$ an ellipticity parameter, which is small compared to unity. With notations of FIG.1(a), $\epsilon$ is equal to $\left(r_{\max }-r_{\min }\right) / 2 a$.

The consequence of this asymmetry is the existence of a vibroacoustic coupling between the acoustic plane wave and the ovalling modes of the structure. Ovalling modes are characterized by a circumferential modal shape in a $\sin (2 \theta)$ form and are often the lowest ones for geometries similar to wind instruments. Physically, a section of the tube is subjected to a uniform pressure distribution due to the plane wave. If the tube is perfectly circular, this tends to dilate and contract it only in a cylindrically symmetric manner, and the section of the tube is only subjected to tension forces. If the tube is oval shaped, then the isotropic pressure distribution implies also bending forces which tend to round the tube by enlarging the small diameter and shortening the bigger one. This movement is directly linked with the ovalling deformation of the pipe. A detailed description of the vibroacoustic coupling is given in appendix A. The conclusion of this appendix is the analytical expression of the acoustic input impedance of the vibrating tube which can be written as :

$$
Z(\omega)=Z_{r}(\omega)(1+C(\omega))
$$

where $C$ is a correction factor describing the wall vibration effect. Considering only the interaction between the internal acoustic pressure and a single ovalling mode, it is shown that :

$$
C(\omega) \propto \frac{\epsilon^{2}}{\left(1-e^{-2 j k L}\right) \cos (k L) m_{\mu}\left(\omega_{\mu}^{2}\left(1-j \eta_{\mu}\right)-\omega^{2}\right)},
$$

where $m_{\mu}$ is the modal mass, which is defined in appendix in Eq.A6, $\omega_{\mu}$ is the modal natural angular frequency and $\eta_{\mu}$ the modal damping, which is the natural decrease rate of the mode. These parameters are obtained experimentally from the modal testing of the tube used in the experiments,(see section III.B.1). Eq.6 allows a direct interpretation of the correction factor. Firstly, the bigger the ellipticity $\epsilon$, the more the input impedance is disturbed. This is due to the increase of the coupling between the ovalling mode and the inner pressure field. 
Secondly, $C$ is inversely proportional to the modal mass $m_{\mu}$, which means that disturbance will be more important when the cylinder is thin and light. Thirdly, when the driving angular frequency $\omega$ approaches the mechanical angular eigenfrequency, the disturbance increases. Finally, at the acoustical resonances of the tube, when $\cos (k L)$ is minimum, the disturbance is maximum. The correction factor $C$ may then take significant values when a frequency coincidence is realized between an acoustical frequency and a mechanical ovalling eigenfrequency.

The value of $\mathrm{C}$ and $\mathrm{Z}$ can be computed. On FIG.2, the modulus of the correction factor $C$ is plotted versus frequency. The regularly spaced peaks correspond to the effect of acoustical resonance and the other peaks correspond to the effects of the resonances of the ovalling modes. FIG.3 represents the modulus of the computed input impedance of the vibrating tube. It shows additional peaks due to the mechanical resonances of ovalling modes.

\section{B. Model of a clarinet like instrument in playing conditions}

In the playing situation, the air column oscillation described by its input impedance is coupled to the mouthpiece and the player. In order to take into account this coupling, the instrument-player system is assimilated to a dynamic system whose behavior can be described by three equations ${ }^{19}$.

a) The linear behavior of the tube resonator is described by the input impedance $\mathrm{Z}$ and the associated Eq.1.

b) The reed is assumed to behave like a single degree of freedom oscillator whose equation of motion is :

$$
\frac{d^{2} h(t)}{d t}+g_{r} \frac{d h(t)}{d t}+\omega_{r}^{2} h(t)=\frac{P_{m}-p(t)}{\mu_{r}}
$$

where $h(t)$ is the dynamic reed displacement, $p(t)$ the acoustic pressure inside the mouthpiece, $g_{r}$ the reed damping factor, $\omega_{r}$ its natural frequency, $\mu_{r}$ its mass over area ratio density, and $P_{m}$ the static pressure in the player's mouth. 
c) The last equation establishes the link between the acoustic volume velocity $u(t)$ through the slit of height $H$ in absence of flow and of effective width $w$, and the pressure difference $P_{m}-p(t):$

$$
\begin{gathered}
u(t)=(H+h(t)) w \sqrt{\frac{2\left|P_{m}-p(t)\right|}{\rho}} \operatorname{sign}\left(P_{m}-p(t)\right) \quad \text { if } \quad\left|P_{m}-p(t)\right| \leq P_{M}, \quad \text { and } \\
u(t)=0 \quad \text { if } \quad\left|P_{m}-p(t)\right| \geq P_{M},
\end{gathered}
$$

where $P_{M}$ is the pressure at which the reed closes the slit. For various sets of input parameters, the non linear system of equation has various types of solutions. Trivial solutions correspond to situations where the equilibrium position of the reed is stable. Acoustic pressure is then null and no sound is produced. Periodic solutions correspond to self-sustained regimes of the system, which are of musical interest. Other types of unstable solutions like unstable periodic regimes can occur, and lead for example to multiphonics or to unwanted regimes in a musical context.

This type of model $^{19}$ is classically used for the simulation of single reed instruments and allows the description of the dynamical behavior of reed instruments, and favor the interpretation of the experimental observations. At the end of the next section, the experimental measurements in playing situation are simulated using this model.

\section{EXPERIMENTS}

\section{A. Dimensioning of experimental system}

Influence of wall vibrations is described by a correction factor $\mathrm{C}$ to input impedance, which is usually very small. As a consequence, no significant effect of wall vibration is generally observed experimentally. This effect can be observed only for particular tubes with specific dimensions. In order to emphasize the possible effects of wall vibrations, the geometrical characteristics and material of a tube whose acoustic input impedance is significantly modified by these vibrations have to be determined. As discussed in the theory explained in section II and developed in appendix, a significant perturbation of the acoustic input impedance is expected if mechanical eigenfrequencies occur in the vicinity of one of the first 
acoustic resonances or anti-resonances. In order to provoke this frequency coincidence, the eigenfrequencies of cylindrical shells are computed. Eigenfrequencies depend on geometrical parameters (thickness $h$, radius $a$ and length $L$ ) and constitutive material parameters (Young's modulus $E$, Poisson's ratio $\nu$ and density $\rho_{s}$ ) of the tube. For tubes whose length is much larger than the radius, the first modes of the shell are usually the ovalling modes. Our study is thus focused on these ovalling modes. Computation of the modal basis of a cylindrical shell is analytically tractable only for simply supported boundary conditions ${ }^{20,21}$. For other boundary conditions, a semi analytical and exact procedure can be implemented to compute eigenfrequencies and modal shapes ${ }^{22}$. Some approximate formulas can also be used $^{23}$. For slender tubes $(L / a>>1)$, the first eigenfrequencies of shells of finite length are close to cut on frequencies $f_{m}^{c}$ of the flexural wave associated with the circumferential index $m \geq 2$ ( $m=2$ for ovalling modes) of infinite cylinders. This frequency is given ${ }^{23}$ by :

$$
f_{m}^{c}=\frac{m\left(m^{2}-1\right)}{4 \pi \sqrt{3} \sqrt{m^{2}+1}} \frac{h}{a^{2}} \sqrt{\frac{E}{\rho_{s}\left(1-\nu^{2}\right)}},
$$

Eq.9 can be used with $m=2$ to estimate the eigenfrequency of the first ovalling mode.

From the acoustical point of view, the computation of acoustical eigenfrequencies of a closedopen cylindrical tube neglecting the viscous and thermal losses and considering a null pressure at the open end can be performed using :

$$
f_{n}=(2 n-1) \frac{c}{4 L},
$$

where $c$ is the speed of sound in air, $L$ the length of the tube and $n$ the acoustical longitudinal index.

As experiments are carried out on a clarinet like instrument, the total length of the system is about $50 \mathrm{~cm}$ and the internal radius is $a=7.5 \mathrm{~mm}$. This gives the following series of acoustical eigenfrequencies for the first five acoustical modes: $170 \mathrm{~Hz}, 510 \mathrm{~Hz}, 850 \mathrm{~Hz}$, $1190 \mathrm{~Hz}, 1530 \mathrm{~Hz}$. In order to satisfy the frequency coincidence, geometrical and material parameters of a tube have to be determined so that the ovalling mode eigenfrequency is close to one of these values. The material chosen is brass, as it is often used for wind instruments. 
The material parameters relative to brass are set to $E=110 \mathrm{GPa}, \rho_{s}=8700 \mathrm{~kg} / \mathrm{m}^{3}$ and $\nu=0.3$. According to Eq.9, the only free parameter is the thickness $h$. A thickness of $h=0.2$ mm gives a frequency of $1630 \mathrm{~Hz}$, which is in the vicinity of the fifth acoustical resonance. The tube is then connected to a rigid slide which makes it possible to vary continuously the acoustical resonance frequencies, thanks to a variable total length of the tube, without changing the fixed mechanical resonance frequencies of the vibrating tube. A vibrating tube and a rigid slide satisfying the dimensioning explained in this section has been machined in order to exhibit the wall vibration effect. This device, which makes it possible to satisfy exactly the frequency coincidence, or on the contrary to avoid it, is connected to a clarinet mouthpiece in an artificial mouth. The vibrating tube is made from brass tubing used in musical instrument making, carefully machined to a thickness of about $0.2 \mathrm{~mm}$. Its length is about $24 \mathrm{~cm}$ and its radius is $7.5 \mathrm{~mm}$. The added lengths of the rigid slide in open position and of the mouthpiece which is inside the artificial mouth make an overall length of about $50 \mathrm{~cm}$. The vibrating tube is then used for the mechanical and acoustical measurements.

\section{B. Mechanical and acoustical measurements}

\section{Experimental modal analysis}

Structural modes of the vibrating tube are determined using standard experimental modal analysis. Eigenfrequencies, mode shapes and damping parameters can be extracted from measured frequency response functions $(\mathrm{FRF})$. The FRF, vibration velocity to force ratios, are computed recording the applied force with an impact hammer, and the vibration velocity signal with a laser vibrometer. The laser vibrometer is fixed on a single point of the structure and the impacts were carried out on different points positioned on a circumference and on two generatrices of the studied tube, as shown in FIG.1(b). An example of FRF is plotted in FIG.4. These measurements give a set of FRF which is used for modal identification. The method used for this identification is the least square complex exponential method (LSCE) ${ }^{24,25}$, which is implemented in the modal analysis software from LMS. The 
results of this modal analysis in terms of modal frequencies, modal damping coefficients and mode shapes are presented in FIG.5.

On the plotted frequency band of FIG.4, six modes have been identified. As shown by their circumferential modal shapes, each mode correspond to an ovalling mode, with a circumferential index $m=2$. They differ by their respective orientations indicated by the angle $\varphi$. Actually, the modes can be classified into three pairs, the modes from each pair having very close eigenfrequencies and the same longitudinal index ( $p=1$ for one node, $p=2$ for two nodes and $p=3$ for three nodes). The duplication of these modes is due to the asymmetry of the cylinder which implies the phenomenon of breaking of modal degeneracy : a mode with a circumferential index $m$ ( $m=2$ for ovalling), and a longitudinal index $p$ splits into two modes with different modal frequencies and circumferential mode shapes differing in rotational angle by $\pi /(2 m)$ theoretically, which is $\pi / 4$ for ovalling modes. Modes are subsequently referred to as triplets $\mu=(m, p, s)$ for an easier identification, where $s$ is the symmetry index ( $s=0$ or $s=1$ ). For example, the second mode of FIG.5 is referred to as the triplet $(2,1,1)$. In order to validate this modal model obtained from the LSCE method, simulated FRF have been computed. On FIG.4, a synthesized FRF is plotted against a measured one. The relative error between the two FRF is only a few percent, which supports the validity of the modal basis. It is also noticeable that the measured value of the first eigenfrequency agrees rather well with the calculated one in section II.A, which means that the dimensioning of the tube was accurate.

It is shown theoretically that these ovalling modes may couple to the inner air column oscillations and alter acoustic input impedance, in the vicinity of their eigenfrequencies. Measurements of input impedances of the vibrating tube are presented in the next section.

\section{Acoustic input impedance measurements}

The device ${ }^{26}$ used for measuring the acoustic input impedances is specially designed in order to impose a carefully calibrated acoustic velocity at the entrance of the tube and to 
measure the resulting pressure using a microphone. The excitation is managed using a microphone cartridge used as the acoustic velocity calibrated source. The brass tube is measured in two configurations. For the first one, the cross section is quasi circular, corresponding to a quasi null ellipticity parameter $\epsilon=0 \%$. For the second one, the tube is made oval by flattening between to small planks so that $\epsilon \approx 8 \%$. The tube is actually perfectly circular at its entrance so that it can be connected to the circular slide ; the ellipticity increase to be maximum at the end of the tube. On FIG.6, measurements corresponding to these two configurations are shown.

It can clearly be seen in FIG.6 that mechanical modes have a strong influence on the input impedance of the tube only if is slightly flattened. Otherwise the coupling between the plane acoustic wave and the ovalling modes does not occur. The good agreement between the measured disturbances of input impedance and the calculated ones in section II.A.2 is also noticeable, see FIG.3 and 6. These disturbance of input impedance may then influence the sound produced by the system in playing conditions. This is investigated in the next sections using an artificial blowing machine.

\section{Study of the self-sustained oscillations of the system in playing conditions}

\section{Experimental set-up}

In order to blow and play the resonator as a musical instrument, the slide-tube device mentioned in section III.A is connected to a clarinet mouthpiece which is put into an artificial mouth. This artificial mouth can produce stable musical sounds for long times, a few minutes or a few hours, allowing to keep the embouchure parameters, for example the mouth pressure $P_{m}$, constant. The set-up, composed of the artificial mouth containing the clarinet mouthpiece, the slide and the vibrating tube, can be described as an "artificially blown slide clarinet".

In order to investigate possible changes of sound quality due to wall vibration, some sound pressure measurements are carried out. The acoustic pressure inside the mouthpiece 
is recorded using a Endevco microphone located in a little hole in the mouthpiece. The external radiated pressure is recorded in the near field in order to avoid parasite reflection from surroundings using a Sennheiser KE-4 microphone. This microphone is kept at a fixed position, $5 \mathrm{~cm}$ from the end of the tube and $1 \mathrm{~cm}$ from axis, in order to avoid problems due to the spatial dependence of the external acoustic field. This microphone was used to record sounds and to perform informal listening tests (see section III.C.2). Pressures are recorded for various playing frequencies obtained by pulling or pushing the slide. As the vibrations of the walls modify the input impedance in the vicinity of the eigenfrequencies of ovalling modes, effects on the acoustic signals are expected when an harmonic of the playing frequency matches one of these mechanical eigenfrequencies. As the resonator is cylindrical, this coincidence can occur on an even harmonic with an acoustical antiresonance and on an odd harmonic with an acoustical resonance. These two configurations are studied in sections III.C.2 and III.C.3 respectively.

The amplitudes and phases of the harmonics of the two pressure signals are recorded using a Stanford SR850 lock-in amplifier. This is performed using the built in lock-in detection algorithm. The lock-in detection was chosen because it has a much better SNR ratio for the values of amplitude and phase of each harmonic of the signal than FFT of recorded sounds.

\section{Acoustic resonance perturbation}

For a particular playing configuration depending on the slide length, the perturbation of input impedance due to wall vibration can be positioned on an acoustical resonance. A perturbation of the self-sustained oscillations occurs when the system is playing on the second periodic regime of oscillation, a musical twelfth above the fundamental regime. This is illustrated on FIG.7, where the input impedance is plotted with the position of the harmonics (fundamental, H2, H3, H4). The third harmonic $H 3$ is expected to be perturbed when the system is played because the mechanical additional peak strongly disturbs the input impedance around this harmonic. The amplitudes of the harmonics of 
the inner pressure signal versus playing frequency are plotted on FIG.8. Each measurement point corresponds to a position of the slide.

It appears that the third harmonic is strongly perturbed for two particular frequency ranges. This corresponds to the influence of two couples of mechanical ovalling modes $(2,1,0)$ at $1612 \mathrm{~Hz},(2,1,1)$ at $1618 \mathrm{~Hz}$ for the playing frequencies around $538 \mathrm{~Hz}$ $(3 * 538=1614 \mathrm{~Hz}$, see FIG.5) and $(2,2,0)$ at $1663 \mathrm{~Hz},(2,2,1)$ at $1676 \mathrm{~Hz}$ for the playing frequencies around $555 \mathrm{~Hz}(3 * 555=1665 \mathrm{~Hz}$, see FIG.5). The other harmonics are also perturbed around these two frequencies. This can be attributed to a non linear coupling between harmonics. Using the complex amplitude of each harmonic, an additive sound synthesis is performed. The fundamental frequency is kept constant for each step, in order to focus attention on varying tone color and not on varying pitch. The informal listening of the results of these synthesis show that these perturbations are audible. Another way to illustrate this tone color modification, is the direct listening of the sounds produced by the system. In the case of frequency coincidence, a slight timbral change is heard when comparing the sound of the pipe when it is grasped by the hand and the sound of the free pipe. The sounds could be easily recognised in a-b type comparison tests. In the other case, when the coincidence is not satisfied, no difference is heard whether the tube is grasped or not.

For this coincidence configuration between mechanical and acoustical resonance, the disturbance is rather important and is reflected not only on the third harmonic as expected but also on other harmonics. This could involve an effect not only on spectral content but also on playing frequency. In order to investigate this effect, the added length of the pipe due to the pulling of the slide was measured precisely for each position of the slide using a calliper rule. These lengths are used to calculate theoretical playing frequencies using Eq.10 adding the length of the tube itself, the equivalent length of the mouthpiece and the length correction due to radiation impedance. A comparison between the computed and 
measured frequencies is performed for two configurations : the free-vibrating tube and the tube with vibration damped. A small effect is pointed out, showing a maximum difference of $1 \mathrm{~Hz}$, when the playing frequency is about $555 \mathrm{~Hz}$. This corresponds to the coincidence of the thirs harmonic $H 3$ with modes $(2,2,0)$ and $(2,2,1)$.

Using the model of a single reed instrument in playing conditions described in section II.B, a simulation based on the experimental set-up characteristics can be performed. The set of three equations is solved using the harmonic balance technique ${ }^{18}$ which is a method developed to determine the periodic response of non linear dynamic systems. The application of this method to the three equation system provides the playing frequency, and the complex values of the amplitudes of the harmonics of the pressure signal.

In order to simulate the variable positions of the slide, the original input impedance is transformed to the new input of the tube, and is computed using the following formula:

$$
Z_{2}=\frac{Z_{1}+j \tan (k \delta L)}{1+j Z_{1} \tan (k \delta L)} .
$$

In Eq.11, $Z_{1}$ is the measured input impedance when the slide is at its minimum length, $k$ is the acoustic wave number and $\delta L$ is the added length due to the pulling of the slide. In this case, $Z_{2}$ represents the input impedance of the system, when the slide is pulled by a length $\delta L$. Small variations of $\delta L$ are used to build a set of input impedances. For each input impedance of this set, the harmonic balance is performed considering that the values of the other input parameters are constant, and set to realistic values $\left(g_{r}=2900 \mathrm{~s}^{-1}\right.$, $\left.\omega_{r}=2 \pi * 3000 \mathrm{rad} / \mathrm{s}, \mu_{r}=0.02 \mathrm{~kg} / \mathrm{m}^{2}, H=1 \mathrm{~mm}, w=1 \mathrm{~cm}, P_{m}=8000 \mathrm{~Pa}\right)$. The results in terms of amplitudes of harmonics and playing frequencies are displayed in FIG.9. This figure shows that the model can describe the perturbation of the third harmonic as expected and already measured. The perturbation of other harmonics due to non linear coupling is also observed. Some important differences between measurements and simulations can be observed showing that the used simplified model is not perfectly accurate. Nevertheless, the disturbance due to wall vibrations can be simulated. The observed differences may have 
various origins. There are a few slight changes in diameter due to the slide, and mechanical parts used to connect the tube, which means that the use of Eq.11 used for the computation of the input impedance for each simulation may be an error source as the bore is not a pure cylinder. Particularly, the position of anti-resonances may not be exactly correct and thus the even harmonics may be simulated with errors. Moreover, the input parameters of the model like the mouth pressure or the height of the slit may be slightly inaccurate and also imply differences between measurements and simulations.

\section{Acoustic antiresonance perturbation by wall vibrations}

Using the slide described in III.C.2, a playing configuration can be found where the perturbation of input impedance due to wall vibrations matches an antiresonance. This can be realized when the system plays on the third periodic regime of oscillation, two octaves and a third above the fundamental regime, for a particular position of the slide. This is illustrated in FIG.10, where the input impedance is plotted with the positions of the harmonics. In such a configuration a perturbation of the second harmonic $H 2$ is expected when the system is played. Small variations of the slide's length allows this coincidence condition to be either satisfied or not.

For each position of the slide, the amplitudes of the harmonics of the measured inner pressure signal versus playing frequency are recorded. Results are given in FIG.11. It is noticeable that the second harmonic $(H 2)$ is perturbed at two particular frequencies corresponding to the influence of two couples of mechanical ovalling modes $(2,1,0),(2,1,1)$ for the playing frequencies around $807 \mathrm{~Hz}(2 * 807=1614 \mathrm{~Hz}$ see FIG.5) and $(2,2,0),(2,2,0)$ for the playing frequencies around $834 \mathrm{~Hz}(2 * 834=1668 \mathrm{~Hz}$ see FIG.5). In this case of a coincidence between a mechanical ovalling resonance and an acoustic antiresonance, spectral changes in the musical sound are also induced.

In the same way as for the case of an acoustic resonance perturbation (section III.C.2), 
the experiment has been simulated numerically using the three equation model. A set of acoustic input impedances corresponding to various positions of the slide is calculated. For each of them, the model is solved using the harmonic balance method. Results are displayed on FIG.12. Significant differences from the measurements are again observed which show the limit of the simplified model, for the same reasons as for section III.C.2. Spectral changes are however fairly well simulated using this model.

\section{DISCUSSION}

In section III.C, for this unusual instrument, it has been shown that some slight tone colour variations can occur when an acoustic resonance or antiresonance coincides with the resonance frequency of an ovalling mode of the resonator. An approximate value of the first ovalling frequency has been computed for real instruments, using realistic geometrical and material parameters. The values, given in table I, differ a lot between various wind instruments. As the eigenfrequency of the first ovalling mode for the flute is $1900 \mathrm{~Hz}$, effects similar to the ones described in the present paper, such as slight tone color modification might occur, but may however be even weaker due to their weak ellipticity and because of additional mechanical damping due to stoppers or finger tips. Acoustic impedance measurements of a real flute resonator has been carried out, but no clear evidence of additional peaks appeared. Brass instrument bells, which have large diameters and low mechanical frequencies ${ }^{28}$ and are free to vibrate, might show small acoustic impedance disturbance. For metal clarinet or trombone slide, coincidence may occur on a high harmonic, and effects are unlikely to be expected. For the wooden clarinet, such effects, even if present, are likely to be inaudible as the ovalling frequency lies in the ultrasonic range. On the contrary, because of their softer construction material (lead tin alloy) and a larger inner radius, greater effects can occur for organ pipes, as the ovalling frequency is only a few hundred $\mathrm{Hz}$ as shown in Table I. This mechanical frequency may match the fundamental playing frequency on particular pipes in a stop. In that case, wall vibrations may even induce transition to pseudo-periodic regimes 
of oscillation and wolf notes as it has been already observed experimentally ${ }^{12}$. To confirm this assumption, input impedance measurements of a real organ pipe resonator have been carried out and showed additional peaks due to mechanical ovalling modes at frequencies of about $300 \mathrm{~Hz}$.

\section{CONCLUSION}

In this paper, effects of wall vibrations on a simplified wind instrument have been investigated considering a coupling between the duct and the internal pressure field. For a very thin and oval shaped tube, with geometrical parameters unusual in comparison to that of real instruments, such a coupling has been demonstrated. Firstly, the theoretical prediction of a coupling between mechanical ovalling mode of a duct with oval cross-section and the plane propagating acoustic mode has been presented and confirmed experimentally. Due to this coupling, the acoustic input impedance is perturbed. The perturbation can be significant near the eigenfrequencies of the ovalling modes of the duct. The importance of asymmetry already noticed ${ }^{1,8}$ and studied ${ }^{17}$, has also been shown experimentally and theoretically; it has been demonstrated that for a perfectly symmetrical tube such a coupling cannot occur. In practice, for real instruments, perfect symmetry is not achievable, due to the presence of side holes or keys for example, but would not be sufficient to achieve measurable coupling with mechanical ovalling modes of the structure.

Secondly, the influence of the modification of input impedance on the sound produced by the tested vibrating tube connected to a clarinet mouthpiece has been investigated experimentally and simulated numerically. The results show that when the eigenfrequency of a mechanical mode matches an acoustic resonance or antiresonance, particular behaviors, different from the perfectly rigid case, are found. In this case of frequency coincidence, the spectral content of sounds is slightly different from the perfectly rigid case. These spectral changes involve an audible tone color modification. An artificial oval shaped and thin walled system has been constructed for these effects to be measurable. For real orchestral 
instruments, although the previously described mechanism is not to exclude completely for a particular instrument, similar effects are thus unlikely to be measurable and, even if they were, would only occur for one or a few notes. Higher mechanical eigenfrequencies (except for the flute), additional vibration damping due to finger tips, stoppers and stronger walls, and low ellipticity weaken the effects shown in this paper. The regular lead-tin organ pipes are exceptions because they have a low ovalling frequencies. Such effects can only occur for pipes whose mechanical eigenfrequency coincides with the fundamental playing frequency or with one of its harmonics.

However, other physical processes could possibly induce wall vibration effects, as all vibroacoustic couplings have not been investigated in this paper.

\section{Acknowledgments}

The authors are grateful to Ruben Picó Vila for fruitful discussions. The authors would also like to thank Serge Collin, Patrick Colas and Emmanuel Brasseur for their technical contributions to this study.

\section{APPENDIX A: COMPUTATION OF THE CORRECTION FACTOR $C$ TO INPUT IMPEDANCE}

The principle of computation of the correction factor $C$ is based upon a perturbation method. Firstly, the vibration velocity field of the walls of the resonator resulting from the inner pressure field, as it would be with no vibration of walls, is computed. The vibration field is then used in order to calculate the new inner pressure field taking into account the inner radiation from the vibrating walls. The input impedance of the vibrating resonator is then computed. This method is a similar approach to the one of Pico and Gautier ${ }^{17}$, but the present one takes into account more realistic mechanical boundary conditions and a more precise description of the mechanical modal basis.

The resonator of the instrument is a pseudo-cylindrical shell, clamped at the entrance and 
free at its end, presenting a slight ellipticity, which can be described using the polar equation $r(\theta)=a(1+\epsilon \cos (2 \theta))$ (see FIG.1(a)). The acoustic boundary conditions are modelled by a uniform acoustic velocity $V_{0}$ at the entrance of the tube and a null pressure at the end. FIG. 1(b) displays the notations of the following description.

The motion of this shell is described by the displacement field $\vec{X}(M)=[u, v, w]^{t}$, which results from the inner acoustic field $p(M)$. In harmonic regime, $e^{j \omega t}$ is implicit and the motion equation can be written in a compact form :

$$
-\rho_{s} h\left[\omega^{2}+\omega_{a}^{2} \mathcal{L}\right] \vec{X}(M)=p(M) \cdot \vec{n},
$$

where $\omega_{a}=\frac{1}{r(\theta)} \sqrt{\frac{E}{\rho_{s}(1-\nu)}}$ is a parameter equalling the ring frequency if $r(\theta)=a, \rho_{s}$ is the density of the material, $E$ its Young modulus, $\nu$ its Poisson's ratio and $h$ the thickness of the shell. $\mathcal{L}$ is the Donnell stiffness operator of the non distorted shell:

$$
\mathcal{L}=\left(\begin{array}{ccc}
a^{2} \frac{\partial^{2} \cdot}{\partial z^{2}}+\frac{1-\nu}{2} \frac{\partial^{2} \cdot}{\partial \theta^{2}} & a \frac{1+\nu}{2} \frac{\partial^{2} \cdot}{\partial z \partial \theta} & \nu a \frac{\partial \cdot}{\partial z} \\
a \frac{1+\nu}{2} \frac{\partial^{2} \cdot}{\partial z \partial \theta} & \frac{\partial^{2} \cdot}{\partial \theta^{2}}+a^{2} \frac{1-\nu}{2} \frac{\partial^{2} \cdot}{\partial z^{2}} & \frac{\partial \dot{\dot{\theta}}}{\partial \theta} \\
-\nu a \frac{\partial \cdot}{\partial z} & -\frac{\partial \cdot}{\partial \theta} & -1-\beta\left(a^{4} \frac{\partial^{4} \cdot \dot{ }}{\partial z^{4}}+a^{2} \frac{\partial^{2}}{\partial z^{2}} \frac{\partial^{2} \cdot}{\partial \theta^{2}}+\frac{\partial^{4} \dot{\partial}}{\partial \theta^{4}}\right) .
\end{array}\right)
$$

The eigenmodes of the non distorted shell $\overrightarrow{\phi_{\mu}}$ in vacuum, which can be referenced using the triplet of indices $\mu=(p, m, s)$, can be written as :

$$
\overrightarrow{\phi_{\mu}}=\left[\begin{array}{c}
U_{p}(z) \sin (m(\theta-\varphi)+s \pi / 2) \\
V_{p}(z) \sin (m(\theta-\varphi)+s \pi / 2) \\
W_{p}(z) \sin (m(\theta-\varphi)+s \pi / 2)
\end{array}\right],
$$

where $U_{p}, V_{p}$ and $W_{p}$ are the longitudinal modal shapes of the mode of axial index $p=$ $1,2, \ldots, \sin (m(\theta-\varphi)+s \pi / 2)$ is the circumferential modal shape with circumferential index $m=0,1,2, \ldots$ and symmetry index $s=0$ or 1 . The angle $\varphi$ represents the direction of the principal axis of the considered mode, which is not necessarily null, as explained by Soedel ${ }^{21}$. Using the expansion over the in vacuo modes $\vec{X}(M)=\sum_{\mu} A_{\mu} \overrightarrow{\phi_{\mu}}$, reporting it in Eq.A1, and projecting it on a mode $\overrightarrow{\phi_{\mu^{\prime}}}$, one can write:

$$
\rho_{s} h \sum_{\mu} A_{\mu} \int_{S} \overrightarrow{\phi_{\mu^{\prime}}} \cdot \overrightarrow{\phi_{\mu}} \frac{\omega_{\mu}^{2}-\omega^{2}}{\omega_{a}^{2}} d S=\int_{S} \frac{\overrightarrow{\phi_{\mu^{\prime}}} \cdot \vec{n} p(M)}{\omega_{a}^{2}} d S .
$$


The $\theta$-dependency of $\omega_{a}$ in the left-hand term of the Eq.A4 is neglected, this means that mechanical modes are not coupled through the ellipticity of the cylinder. It is also considered that the exciting pressure $p(z)$ is the acoustic field in the case of perfectly rigid walls assuming the light fluid approximation. Eq.A4 can then be written:

$$
A_{\mu} m_{\mu}\left(\omega_{\mu}^{2}-\omega^{2}\right)=\int_{0}^{L} \int_{0}^{2 \pi}{\overrightarrow{\phi_{\mu}}}^{t} \cdot \vec{n} p(z)(1+\epsilon \cos (2 \theta))^{2} r(\theta) d z d \theta
$$

where $\vec{n}$ is the radial vector so that $\vec{\phi}_{\mu}^{t} \cdot \vec{n}=W_{p}(z) \sin (m(\theta-\varphi)+s \pi / 2)$, and $\mu$ the modal mass is defined by

$$
m_{\mu}=\int_{S} \rho_{S} h \vec{\phi}_{\mu}^{t} \cdot \overrightarrow{\phi_{\mu}} d S
$$

The pressure field inside the rigid tube is expressed as :

$$
p(z)=j \rho_{0} c_{0} V_{0} \frac{\sin (k(L-z))}{\cos (k L)} .
$$

Using these expressions, considering that $\epsilon \ll 1$ and introducing the parameter $\eta_{\mu}$ modelling the structural modal damping, the unknown modal amplitudes $A_{\mu}$ are given by :

$$
\begin{gathered}
A_{\mu} m_{\mu}\left(\omega_{\mu}^{2}\left(1-j \eta_{\mu}\right)-\omega^{2}\right)= \\
\frac{j \rho_{0} c_{0} V_{0} a}{\cos (k L)} \int_{0}^{L} W_{p}(z) \sin (k(L-z)) d z \int_{0}^{2 \pi} \sin (m(\theta-\varphi)+s \pi / 2)(1+3 \epsilon \cos (2 \theta)) d \theta,
\end{gathered}
$$

where $\rho_{0}$ is the air density, $c_{0}$ the speed of sound and $V_{0}$ the uniform acoustic velocity at the entrance of the cylinder. This expression shows that if $m=2$ (ovalling mode) and $\epsilon=0$ (perfectly circular shell) the modal amplitudes are null and thus the coupling cannot occur. The two integrals of Eq.A8 are noted as following:

$$
\begin{gathered}
I_{p}=\int_{0}^{L} W_{p}(z) \sin (k(L-z)) d z \\
I_{m}=\int_{0}^{2 \pi} \sin (m(\theta-\varphi)+s \pi / 2)(1+3 \epsilon \cos (2 \theta)) d \theta .
\end{gathered}
$$

For example, for a simply supported shell $W_{p}(z)$ is $W_{p}(z)=\sin (p \pi / L)$. For other boundary conditions the integral can be calculated using an experimental axial modal profile $W_{p}(z)$, which has been done in this paper. Knowing $W_{p}(z)$, the z-axis mode shape which is fitted using a polynomial, $A_{\mu}$ are known and thus the vibrations of the shell is known. 
Using this velocity vibration field, the new inner pressure is then calculated using the integromodal approach. The Green function of the infinite cylinder, considering only the plane wave is:

$$
G\left(z, z_{0}\right)=\frac{e^{-j k\left|z-z_{0}\right|}}{2 j \pi a^{2} k}
$$

The integral representation of the acoustic field in the tube with vibrating walls is:

$$
p(z)=\int_{S} G\left(z, z_{0}\right) \partial_{n} p\left(z_{0}\right)-p\left(z_{0}\right) \partial_{n} G\left(z, z_{0}\right) d S_{0}
$$

On the entrance surface of the cylinder, we have

$\partial_{n} G=\frac{-e^{-j k z}}{2 a^{2} \pi} ; p(0)=P_{0}$ and $\partial_{n} p=j \omega V_{0} \rho_{0}$.

At the end surface of the cylinder :

$\partial_{n} G=\frac{-e^{j k(L-z)}}{2 a^{2} \pi} ; p(L)=0$ and $\partial_{n} p=-j \omega V_{L} \rho_{0}$.

On the lateral surface :

$\partial_{n} G=0 ; \partial_{n} p=-j \rho_{0} \omega V$ and $V=\dot{w}=j \omega w$.

Developing Eq.A11 it is possible to write :

$$
p(z)=\left[B^{+}+D^{+}(z)\right] e^{-j k z}+\left[B^{+}+D^{+}(z)\right] e^{-j k(L-z)},
$$

where functions $D^{+}$and $D^{-}$take into account the vibrations of the walls. $B^{+}, B^{-}, D^{+}$and $D^{-}$are then written as :

$$
\begin{gathered}
B^{+}=\frac{1}{1+e^{-2 j k L}}\left(\rho_{0} c_{0} V_{0}+D^{-}(0) e^{-j k L}-D^{+}(L) e^{-2 j k L}\right), \\
B^{-}=\frac{1}{1+e^{-2 j k L}}\left(-\rho_{0} c_{0} V_{0} e^{-j k L}-D^{-}(0) e^{-2 j k L}-D^{+}(L) e^{-j k L}\right), \\
D^{+}(z)=\frac{-j \rho_{0} c_{0} \omega}{2 a \pi} \sum_{\mu} A_{\mu}\left(\int _ { 0 } ^ { 2 \pi } \operatorname { s i n } ( m ( \theta _ { 0 } - \varphi ) + s \pi / 2 ) \left(1+\epsilon \cos \left(2 \theta_{0}\right) d \theta_{0} \int_{0}^{z} e^{j k z_{0}} W_{p}\left(z_{0}\right) d z_{0},\right.\right. \\
D^{-}(z)=\frac{-j \rho_{0} c_{0} \omega}{2 a \pi} \sum_{\mu} A_{\mu}\left(\int _ { 0 } ^ { 2 \pi } \operatorname { s i n } ( m ( \theta _ { 0 } - \varphi ) + s \pi / 2 ) \left(1+\epsilon \cos \left(2 \theta_{0}\right) d \theta_{0} \int_{z}^{L} e^{-j k\left(z_{0}-L\right)} W_{p}\left(z_{0}\right) d z_{0} .\right.\right.
\end{gathered}
$$

The modal amplitudes are known using Eq.A8, and the integrals can be calculated knowing $W_{p}(z)$. The integrals of Eq.A13 are noted :

$$
\begin{gathered}
J_{p}=\int_{0}^{L} e^{j k z_{0}} W_{p}\left(z_{0}\right) d z_{0} \\
K_{p}=\int_{0}^{L} e^{-j k\left(z_{0}-L\right)} W_{p}\left(z_{0}\right) d z_{0}, \\
I_{m}^{\prime}=\int_{0}^{2 \pi} \sin \left(m\left(\theta_{0}-\varphi\right)+s \pi / 2\right)\left(1+\epsilon \cos \left(2 \theta_{0}\right) d \theta_{0} .\right.
\end{gathered}
$$


Using Eq.A12, the input impedance is :

$$
Z=\frac{P_{0}}{V_{0}}=\frac{B^{+}+\left(B^{-}+D^{-}(0)\right) e^{j k L}}{V_{0}}
$$

After some algebra using Eq.A8 and Eq.A13, it is possible to write the input impedance as

$$
Z=Z_{r}(1+C)
$$

where $Z_{r}=j \rho_{0} c_{0} \tan (k L)$ is the input impedance of the rigid cylinder and $C$ a correction factor to this input impedance which is equal to

$$
C=\frac{2 e^{-j k L}}{\rho_{0} c_{0}\left(1-e^{-2 j k L}\right)} \frac{D^{-}(0)}{V_{0}}-\frac{2 e^{-2 j k L}}{\rho_{0} c_{0}\left(1-e^{-2 j k L}\right)} \frac{D^{+}(L)}{V_{0}}
$$

where

$$
\begin{aligned}
& D^{+}(L)=\sum_{\mu} D_{\mu}^{+}(L)=\sum_{\mu} \frac{\rho_{0}^{2} c_{0}^{2} V_{0} \omega I_{m} I_{m}^{\prime} I_{p} J_{p}}{2 \pi \cos (k L) m_{\mu}\left(\omega_{\mu}^{2}\left(1-j \eta_{\mu}\right)-\omega^{2}\right)} \\
& D^{-}(0)=\sum_{\mu} D_{\mu}^{+}(L)=\sum_{\mu} \frac{\rho_{0}^{2} c_{0}^{2} V_{0} \omega I_{m} I_{m}^{\prime} I_{p} K_{p}}{2 \pi \cos (k L) m_{\mu}\left(\omega_{\mu}^{2}\left(1-j \eta_{\mu}\right)-\omega^{2}\right)} .
\end{aligned}
$$

For a single ovalling mode of index $\mu=(2, p, s)$, the coefficient $C$ is proportional to

$$
\frac{\epsilon^{2}}{\left(1-e^{-2 j k L}\right) \cos (k L) m_{\mu}\left(\omega_{\mu}^{2}\left(1-j \eta_{\mu}\right)-\omega^{2}\right)} \text {. }
$$

\section{REFERENCES}

1 N. H. Fletcher and T. D. Rossing, The physics of musical instruments, (Springer; Second edition, 2005)

2 C. J. Nederveen, Acoustical aspects of woodwind instruments, (Northern Illinois University Press, Rev Sub edition, 1998).

3 A. H. Benade, Fundamentals of musical acoustics, (Dover Publications, Reprint edition 1990).

4 R. Smith, "The effect of material in brass instruments; a review", Proceedings of The Institute of Acoustics 8(1), 91-96, (1986).

5 D. C. Miller, "The influence of the material of wind-instruments on the tone quality", Science 29(735), 161-171, (1909). 
${ }^{6}$ V. C. Mahillon, Eléments d'acoustique musicale et instrumentale ("Elements of musical and instrumental acoustics"), Manufacture générale d'instruments de musique, Bruxelles, (1874).

7 F. Gautier and N. Tahani, "Vibroacoustic behavior of a simplified musical wind instrument", J. Sound. Vib. 213(1), 107-125, (1998).

8 P. S. Watkinson and J. M. Bowsher, "Vibration characteristics of brass instrument bells", J. Sound. Vib. 85(1), 1-17, (1982).

9 J. Whitehouse, A study of the wall vibrations excited during the playing of lip-reed instruments, (Ph. D. Thesis, Technology Faculty, The Open University, 2003).

10 J. Backus, "Effect of wall material on the steady-state tone quality of woodwind instruments", J. Acoust. Soc. Am. 36(10), 1881-1887 (1964).

11 S. N. Yousri and F. J. Fahy "Distorted cylindrical shell response to internal acoustic excitation below the cut-off frequency", J. Sound. Vib. 52(3), 441-452, (1977).

12 C. J. Nederveen and J. P. Dalmont," Pitch and level changes in organ pipes due to wall resonances", J. Sound. Vib. 271(1-2), 227-239 (2004).

13 J.-P. Dalmont, J. Gilbert and S. Ollivier, "Nonlinear characteristics of single reed instruments: Quasistatic volume flow and reed opening measurements", J. Acoust. Soc. Am. $\mathbf{1 1 4}(4), 2253-2262(2003)$.

14 J. Backus, "Input impedance curves for the reed woodwind instruments", J. Acoust. Soc. Am. 56(4), 1266-1279 (1974).

15 J. P. Dalmont, B. Gazengel, J. Gilbert and J. Kergomard, "Some aspects of tuning and clean intonation in reed instruments", Applied Acoustics 46, 19-60 (1995).

16 M. Bruneau, Manuel d'acoustique fondamentale ("Fundamentals of acoustics"), (Edition HERMES, Paris, 1998), p.145.

17 R. Picó Vila and F. Gautier, "The vibroacoustics of slightly distorted cylindrical shells: A model for acoustic input impedance", J. Sound. Vib. 302(2), 18-38 (2007).

18 J. Gilbert, J. Kergomard and E. Ngoya, "Calculation of the steady state oscillations of a clarinet using the harmonic balance technique", J. Acoust. Soc. Am. 86(1), 35-41 (1989). 
J. Kergomard, Elementary considerations on reed-instruments oscillations, In: Mechanics of Musical Instruments. A. Hirschberg, et al., editors. Lecture notes. CISM. (Springer Verlag, 1995) p. 229290

20 A. W. Leissa, Vibration of shells, (Acoustical Society of America, 1993).

21 W. Soedel, Vibration of shells and plates, (Marcel Dekker, New York, 1981).

22 D. F. Vronay and B. L. Smith, "Free vibration of circular cylindrical shells of finite length", A.I.A.A. Journal 8(3), 601-603 (1970).

23 R. D. Blevins, Formulas for natural frequency and mode shape, (Krieger Publishing Company 1979).

24 D. J. Ewins, Modal testing: theory, practice and application, (Taylor and Francis Group, second edition, 2001).

25 J. Piranda, "Analyse modale expérimentale" ("Experimental modal analysis"), Techniques de l'ingénieur, R6(180), 1-29 (2001).

26 J. P. Dalmont and A. M. Bruneau, "Acoustic impedance measurements: plane-wave mode and first helical mode contributions", J. Acoust. Soc. Am. 91, 3026-3033 (1992).

27 G. Nief, J.-P. Dalmont, F. Gautier and J. Gilbert, "Influence des vibrations de parois d'un tuyau sur son impédance d'entrée : application aux instruments à vent" ("Influence of wall vibrations of a pipe on its input impedance : application to wind instruments"), Actes du 8ème Congrès Français d'Acoustique, Tours (2006).

28 T. R. Moore, J. D. Kaplon, G. D. McDowall and K. A. Martin, "Vibrational modes of trumpet bells", J. Sound. Vib. 254(4), 777-786 (2002). 


\begin{tabular}{|c||c|c|c|c|c|}
\hline \hline & $\begin{array}{c}\text { Ebony Bb } \\
\text { clarinet }\end{array}$ & $\begin{array}{c}\text { Metal Bb } \\
\text { clarinet }\end{array}$ & Organ pipe & Flute & Trombone slide \\
\hline Thickness (mm) & 5 & 0.5 & 0.5 & 0.35 & 0.5 \\
Internal radius (mm) & 7 & 7 & 25 & 9.5 & 7.5 \\
Length (m) & 0.5 & 0.5 & 0.6 & 0.6 & 2.5 \\
\hline Young's modulus (GPa) & 3 & 110 & 35 & 125 & 110 \\
Poisson's ratio & 0.35 & 0.33 & 0.33 & 0.33 & 0.33 \\
Density (kg/m $\left.{ }^{3}\right)$ & 1000 & 8500 & 8700 & 8700 & 8500 \\
\hline First ovalling mode & 23000 & 4800 & 210 & 1900 & 4200 \\
frequency (Hz) & & & & & \\
\hline \hline
\end{tabular}

TABLE I. Approximate value of the frequency of the first ovalling mode for wind instruments. 


\section{LIST OF FIGURES}

FIG. 1 (a)Polar graph of the oval cross section of the tube

(b) Notations and coordinates systems used in appendix for the pseudo cylindrical shell, Position of the measured points on the tested tube for modal

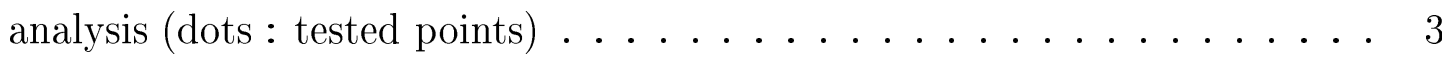

FIG. 2 Calculated correction factor C of a brass tube, $24 \mathrm{~cm}$ long, $0.2 \mathrm{~mm}$ thick, $7.5 \mathrm{~mm}$ radius, with a $8 \%$ ellipticity. (a) broad frequency band ; (b) zoom (Modulus in dB scale) $\ldots \ldots \ldots \ldots \ldots$

FIG. 3 Calculated acoustic input impedance of a brass tube, $24 \mathrm{~cm} \mathrm{long,} 0.2 \mathrm{~mm}$ thick, $7.5 \mathrm{~mm}$ radius, with a $8 \%$ ellipticity. (a) broad frequency band ;(b) zoom (Modulus in dB scale)

Perfectly rigid tube (dotted line) : peaks correspond to acoustical resonances Vibrating tube (solid line) : additional peaks correspond to mechanical ovalling modes resonances $\ldots \ldots \ldots \ldots \ldots$

FIG. 4 Measured FRF (solid line) and synthesised FRF using the parameters obtained from modal analysis (dotted line) for the thin brass tube . . . . . .

FIG. 5 Modal parameters (eigenfrequencies and dampings) of the brass tube, Longitudinal modal shape : measurements (dotted line), fit (solid line) and Circumferential modal shapes . . . . . . . . . . . . . .

FIG. 6 Measured input impedance of the thin brass tube: (a) broad frequency band

; (b) zoom on the frequency band containing mechanical modal frequencies. (Modulus in dB scale)

Quasi-cylindrical tube (dotted line) : no coupling with mechanical modes Slightly flattened tube (solid line) : coupling with mechanical modes . . . 
FIG. 7 Input impedance of the vibrating tube connected to the slide and to the mouthpiece when the frequency coincidence between the third harmonic $H 3$ and the mechanical eigenfrequency of the $(2,2,0)$ ovalling mode is satisfied. Frequencies of harmonics $H 1, H 2$ and $H 3$ are multiple of the playing frequency $F_{0} \ldots \ldots \ldots \ldots \ldots \ldots \ldots \ldots \ldots \ldots \ldots \ldots \ldots \ldots \ldots$

FIG. 8 Amplitude of harmonics of the inner pressure signal as a function of playing frequency $($ measurements) . . . . . . . . . . . . . 37

FIG. 9 Amplitude of harmonics of the inner pressure signal as a function of playing frequency $($ harmonic balance simulation $) \ldots \ldots . \ldots 38$

FIG. 10 Input impedance of the vibrating tube connected to the slide and to the mouthpiece when the frequency coincidence between the second harmonic $\mathrm{H} 2$ and the mechanical eigenfrequency of the $(2,2,0)$ ovalling mode is satisfied. Frequencies of harmonics $H 1, H 2$ are multiple of the playing frequency $F_{0}$.

FIG. 11 Amplitude of harmonics of the inner pressure signal as a function of playing frequency (measurements) . . . . . . . . . . . . . 40

FIG. 12 Amplitude of harmonics of the inner pressure signal as a function of playing

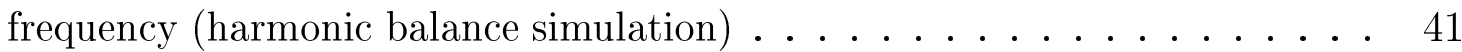




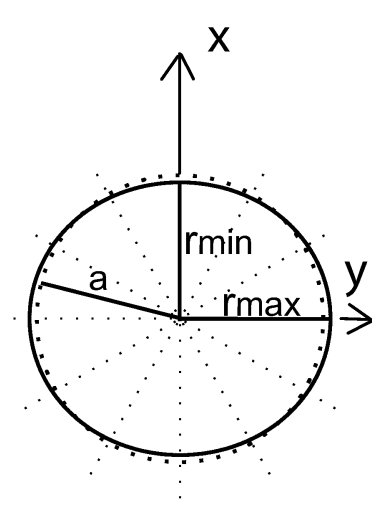

(a)

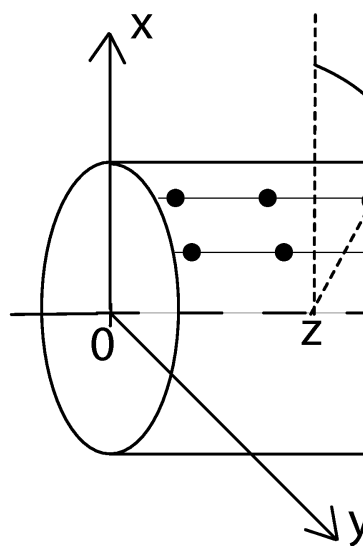




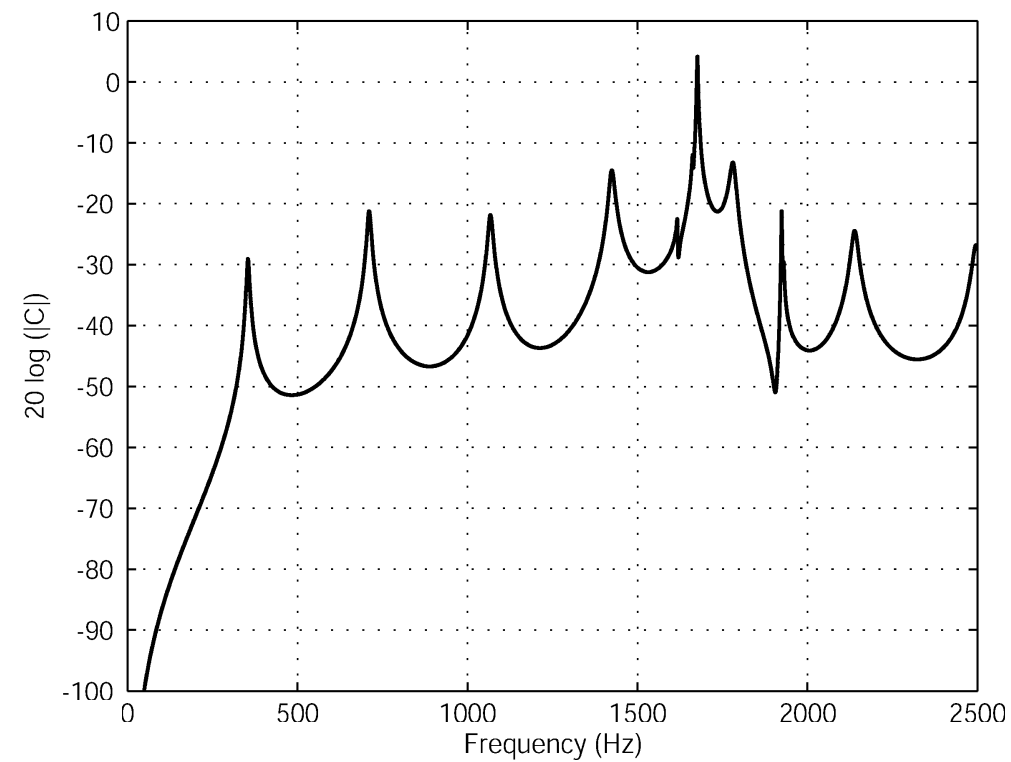

(a)

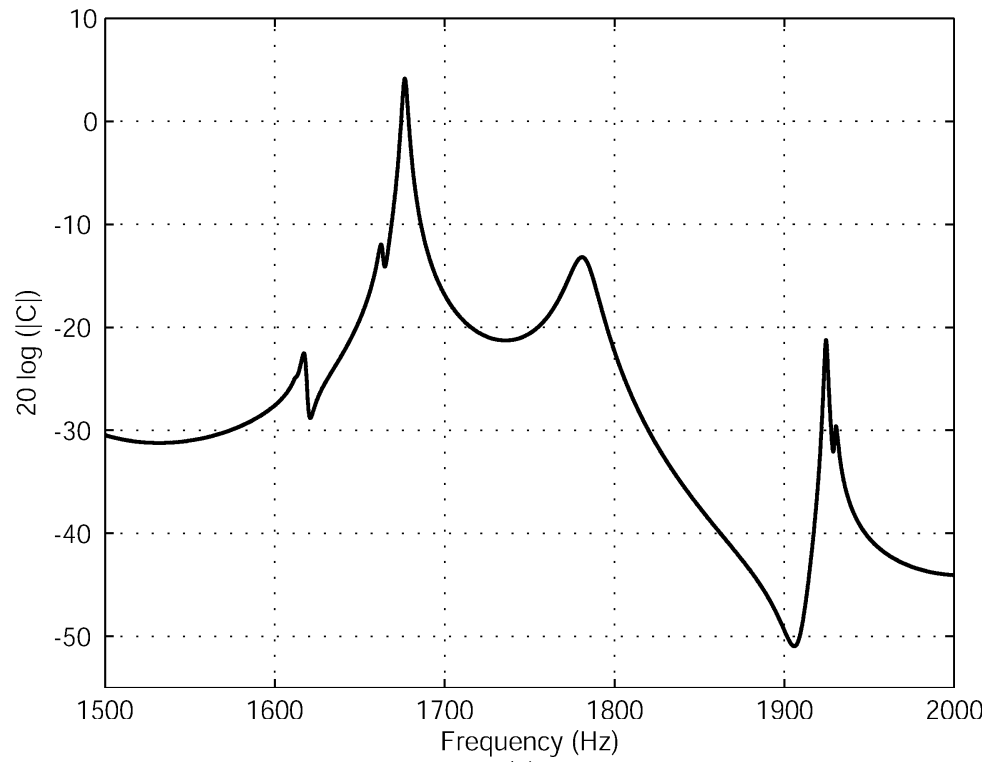

(b)

FIG. 2. 


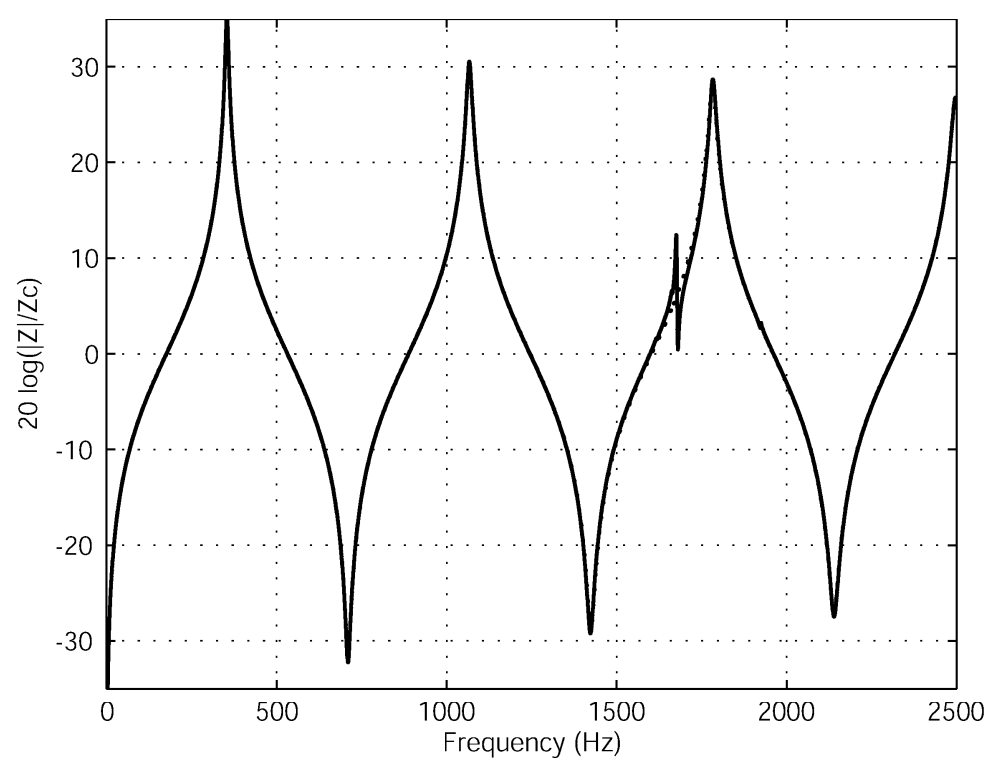

(a)

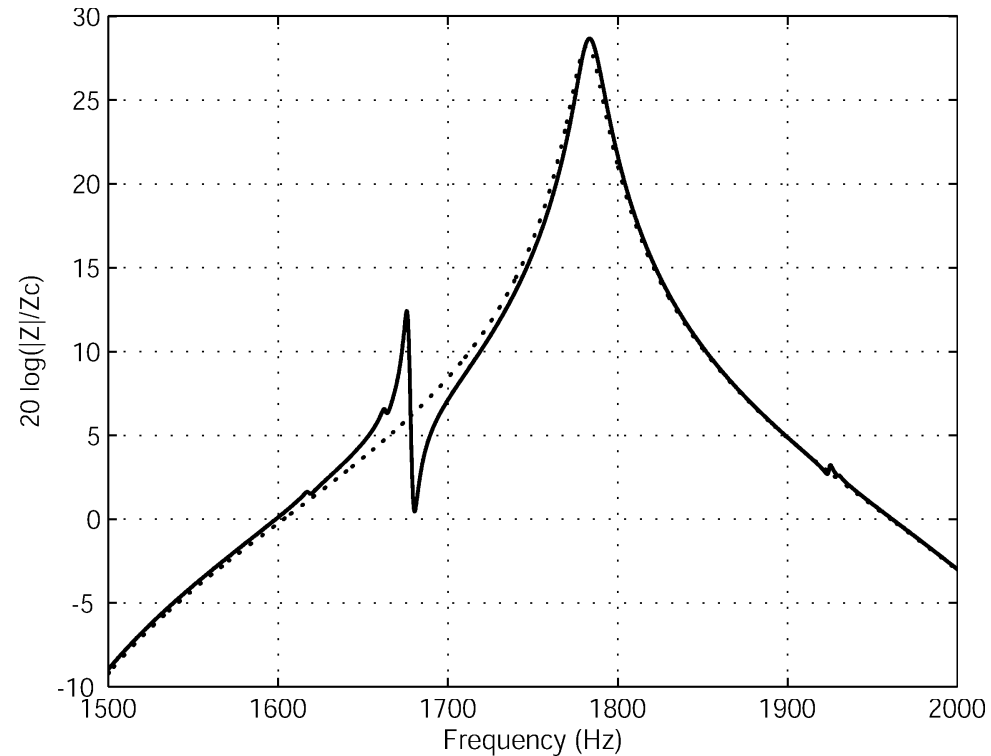

(b)

FIG. 3. 


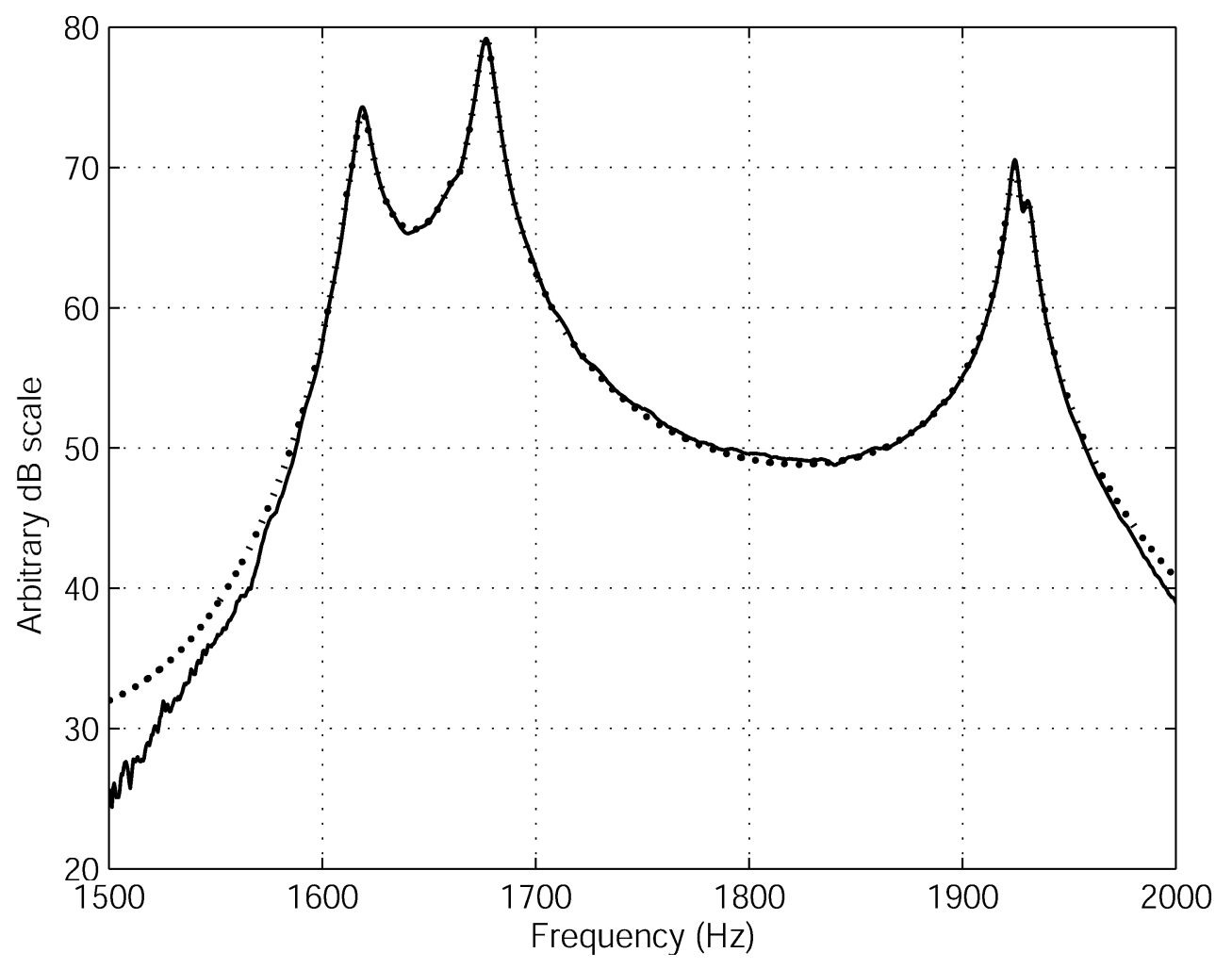

FIG. 4. 


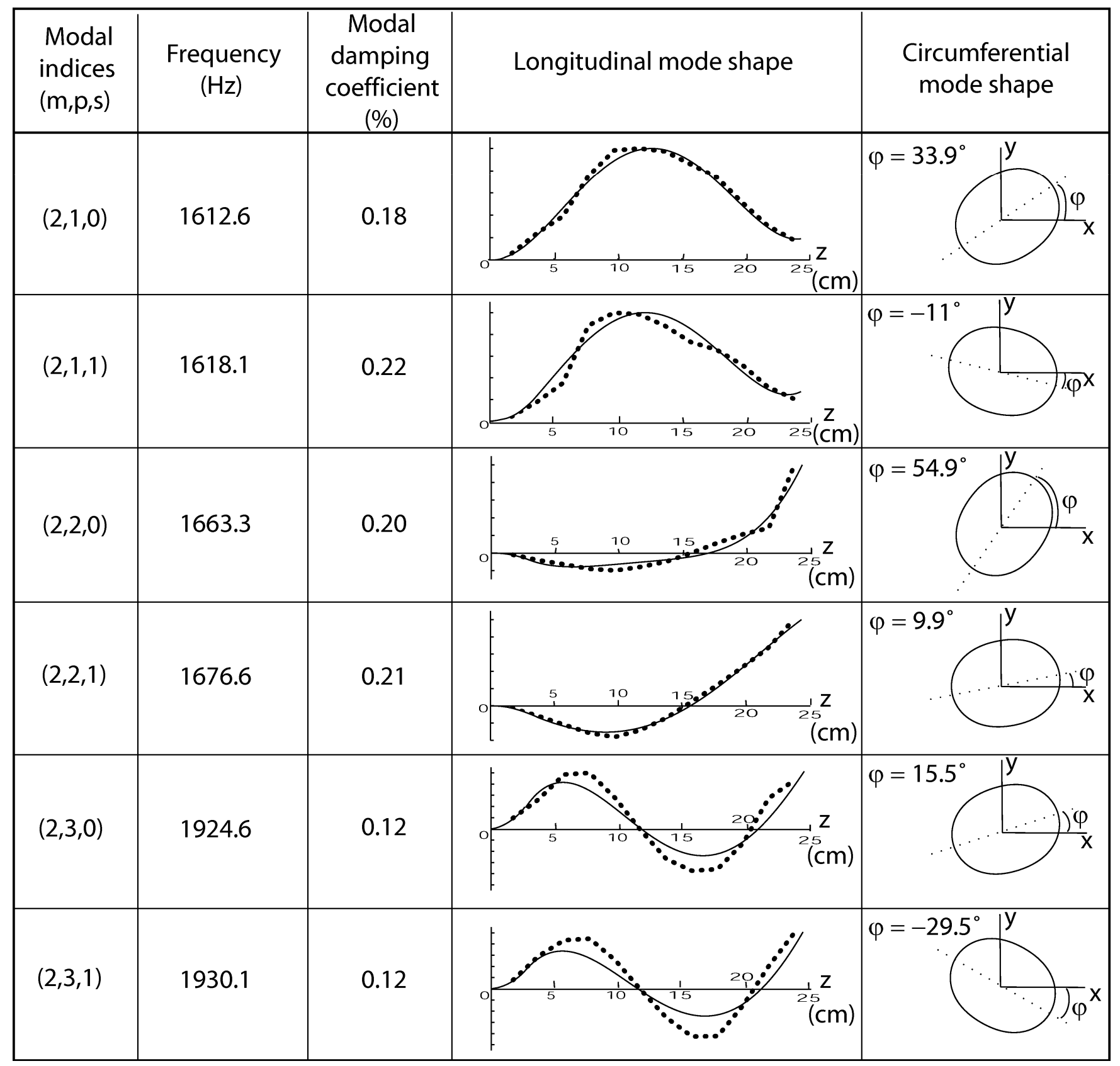

FIG. 5. 


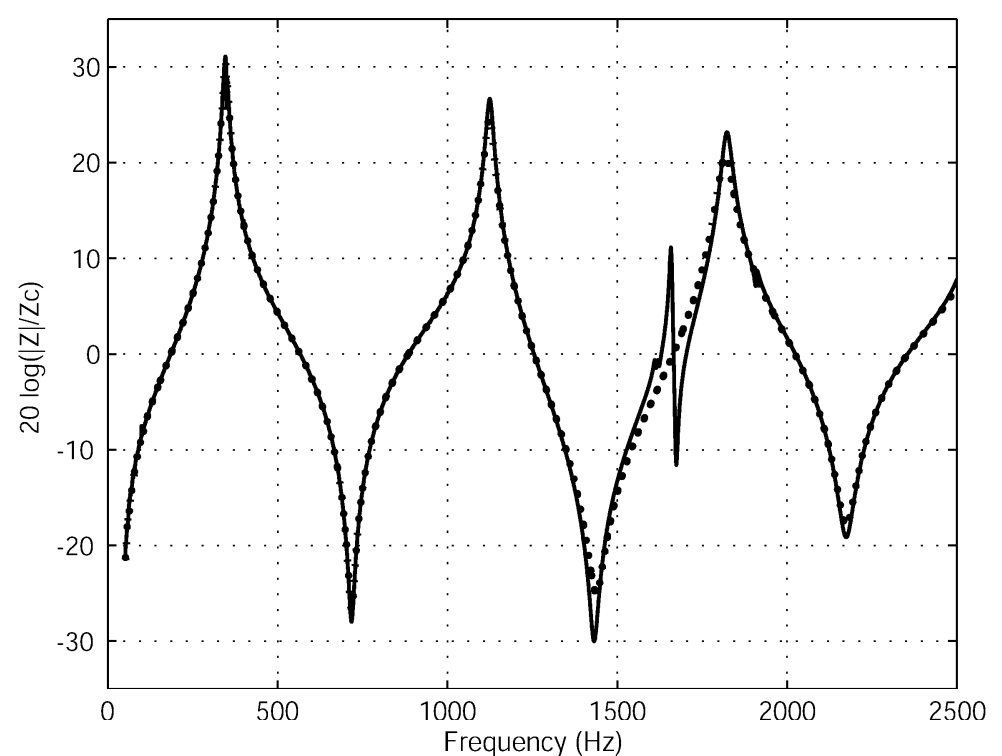

(a)

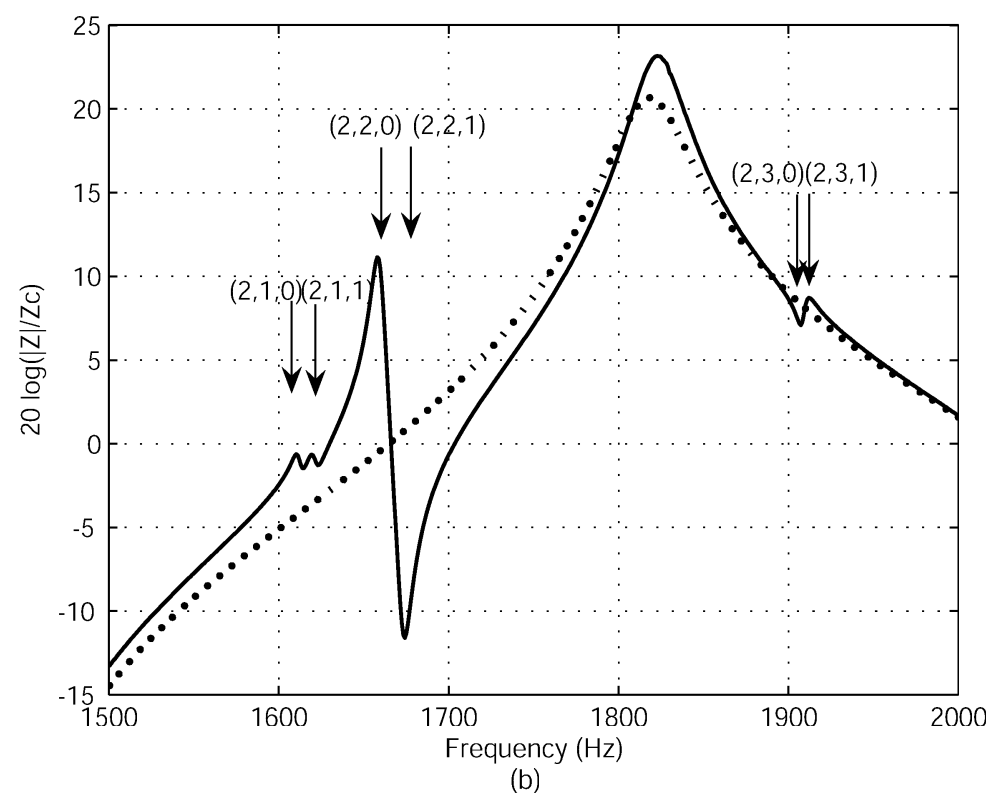

FIG. 6. 


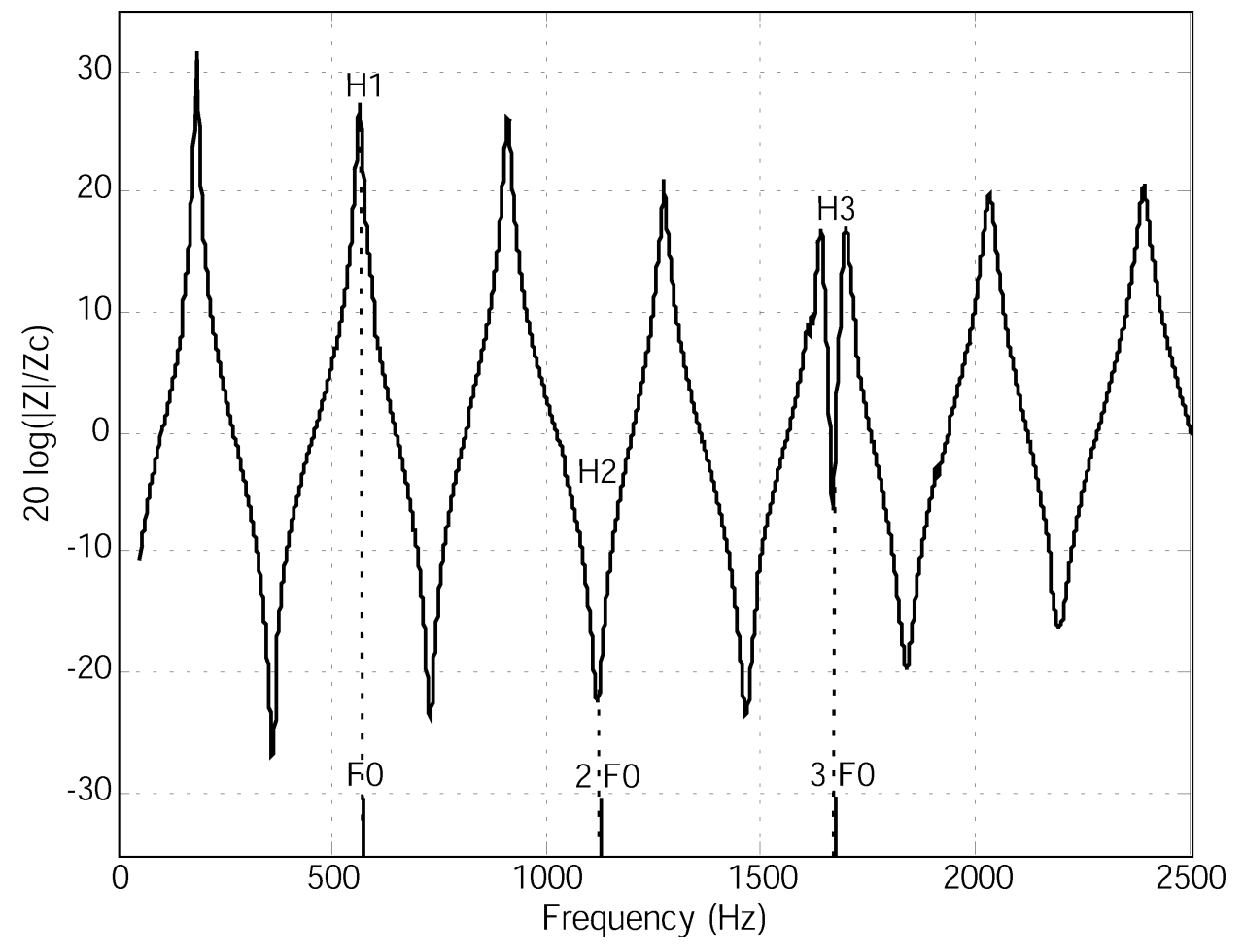

FIG. 7. 


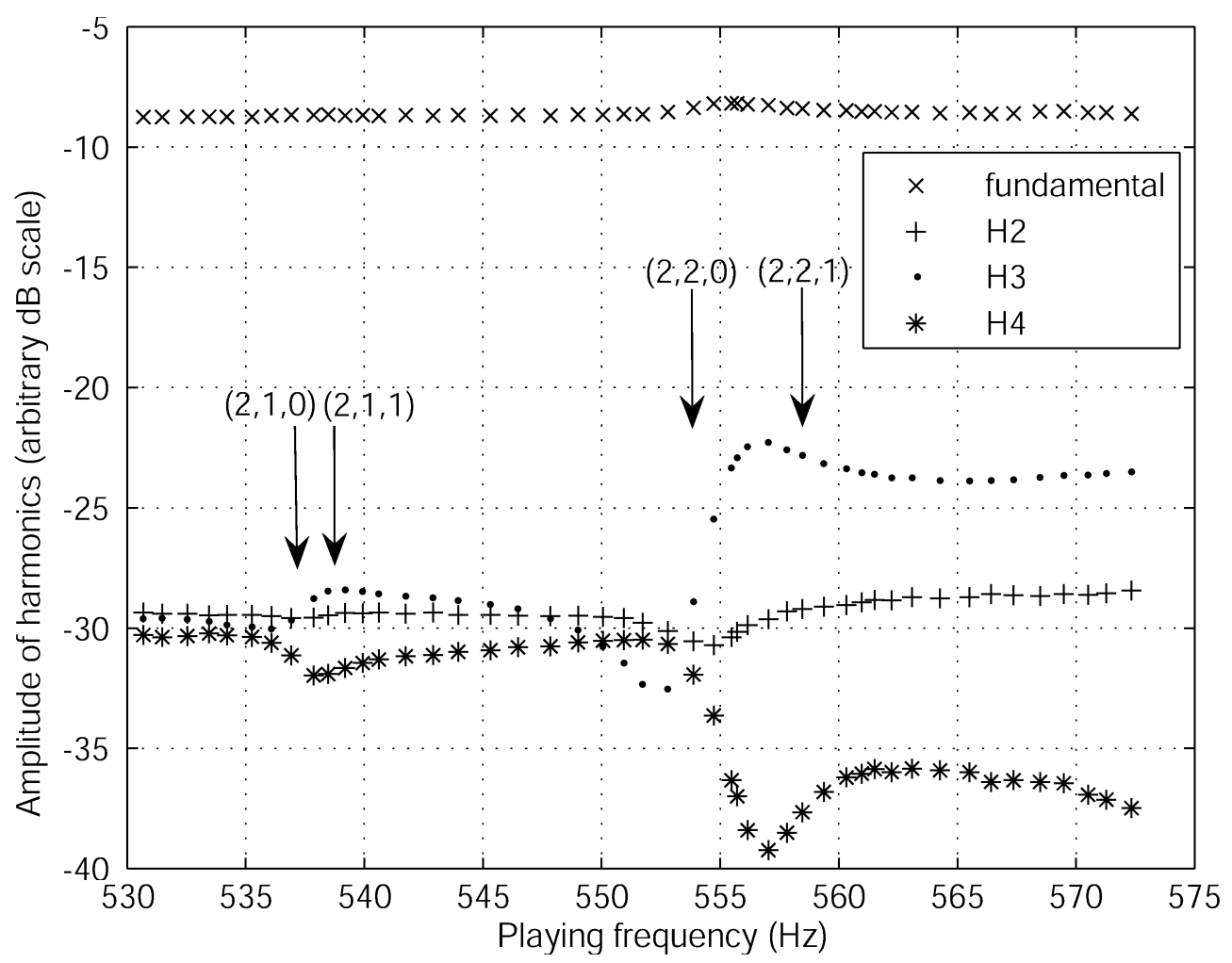

FIG. 8. 


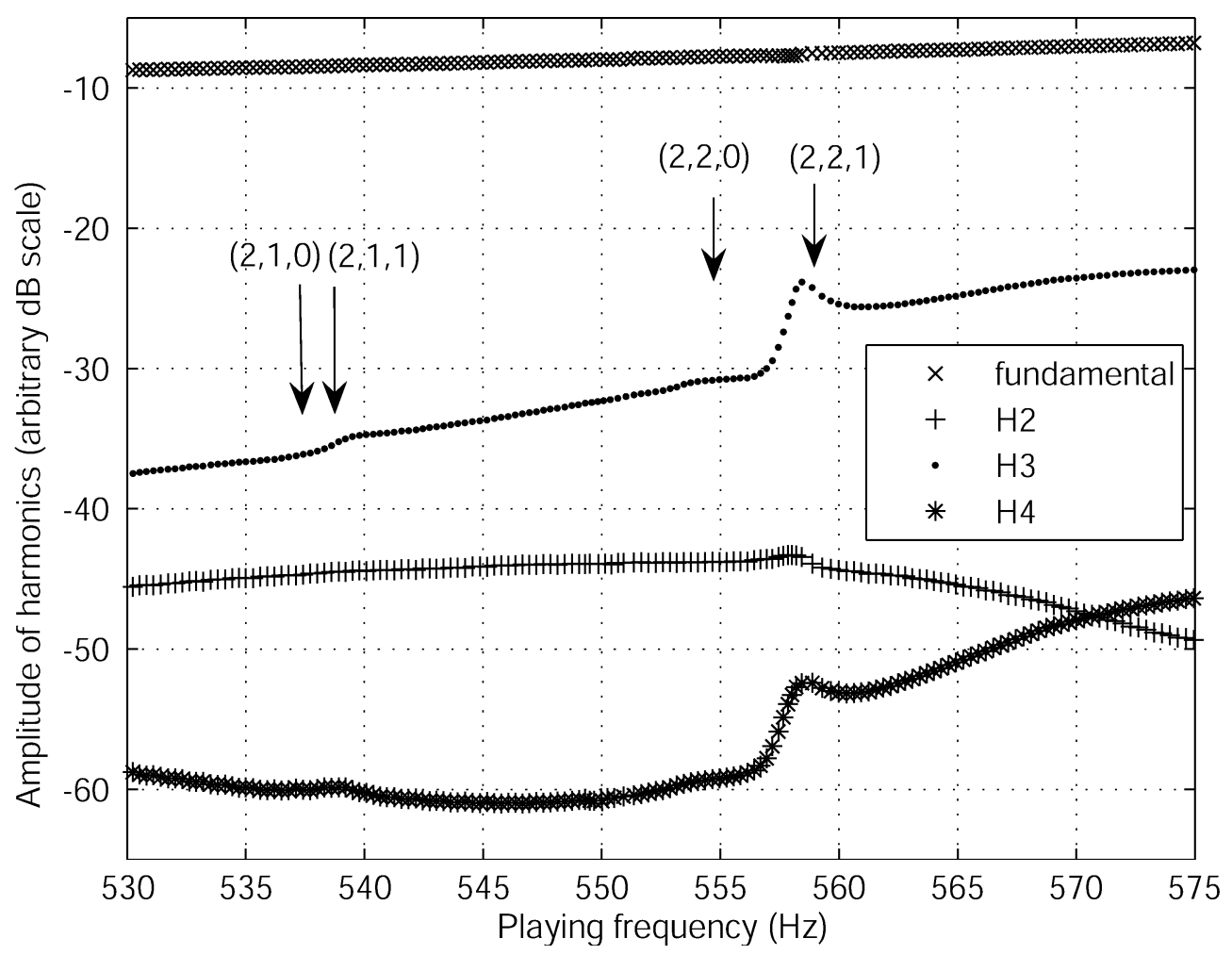

FIG. 9. 


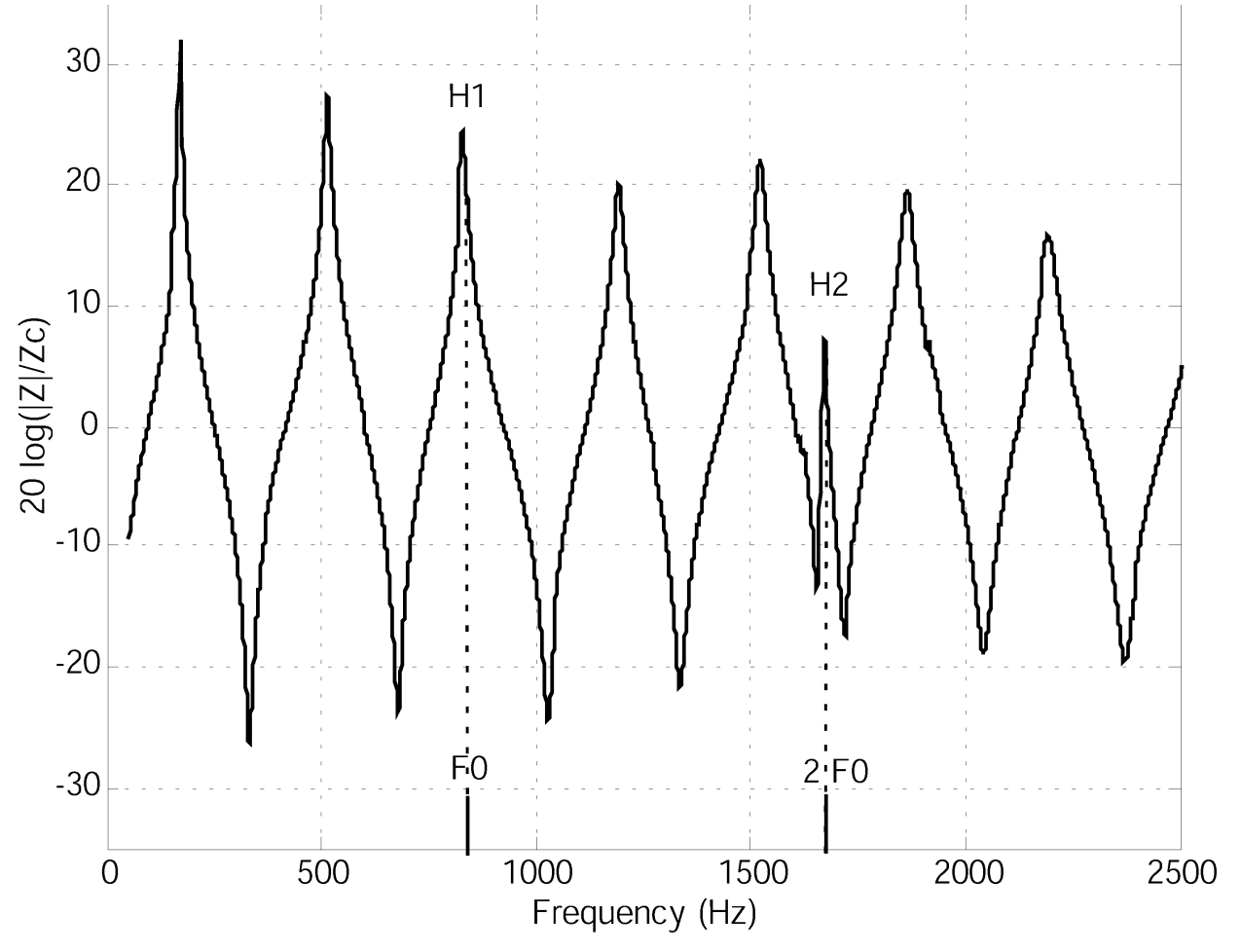

FIG. 10. 


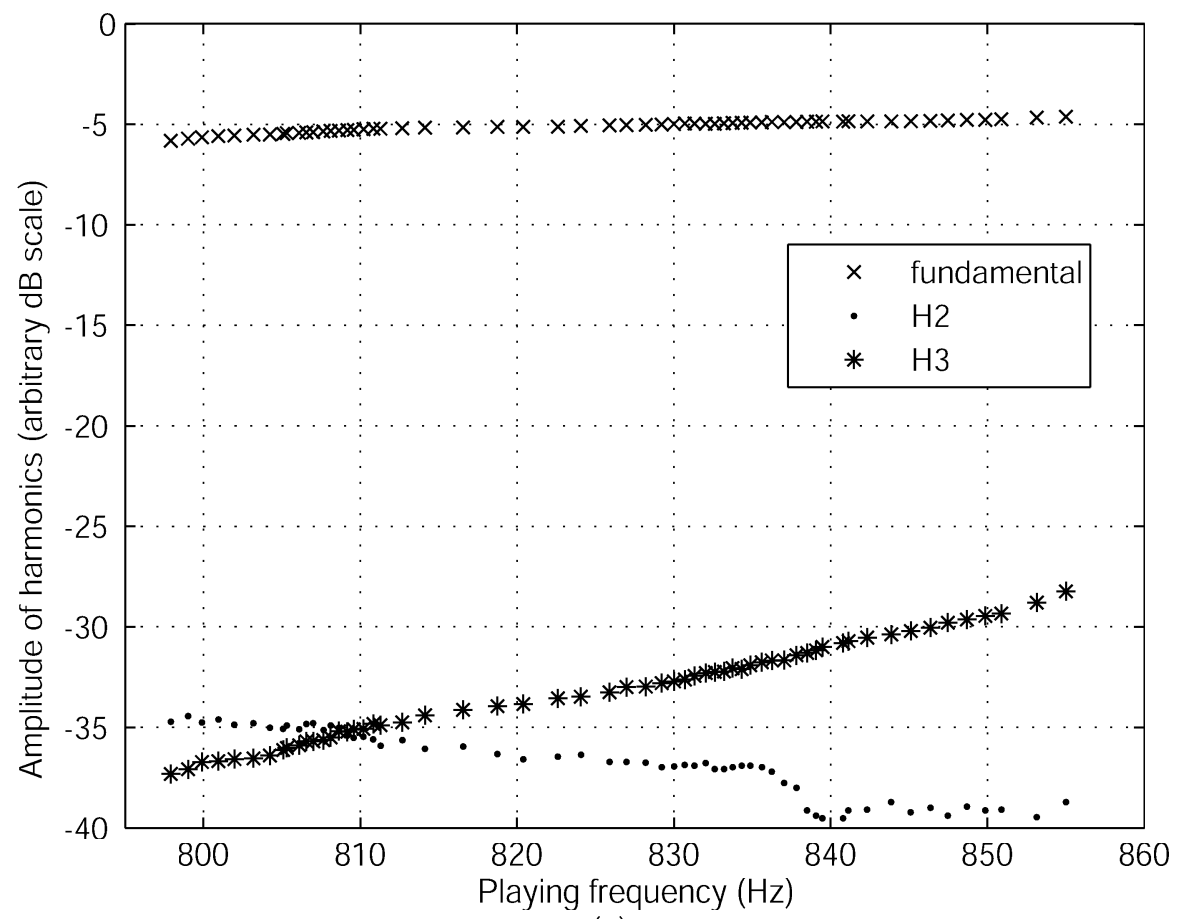

(a)

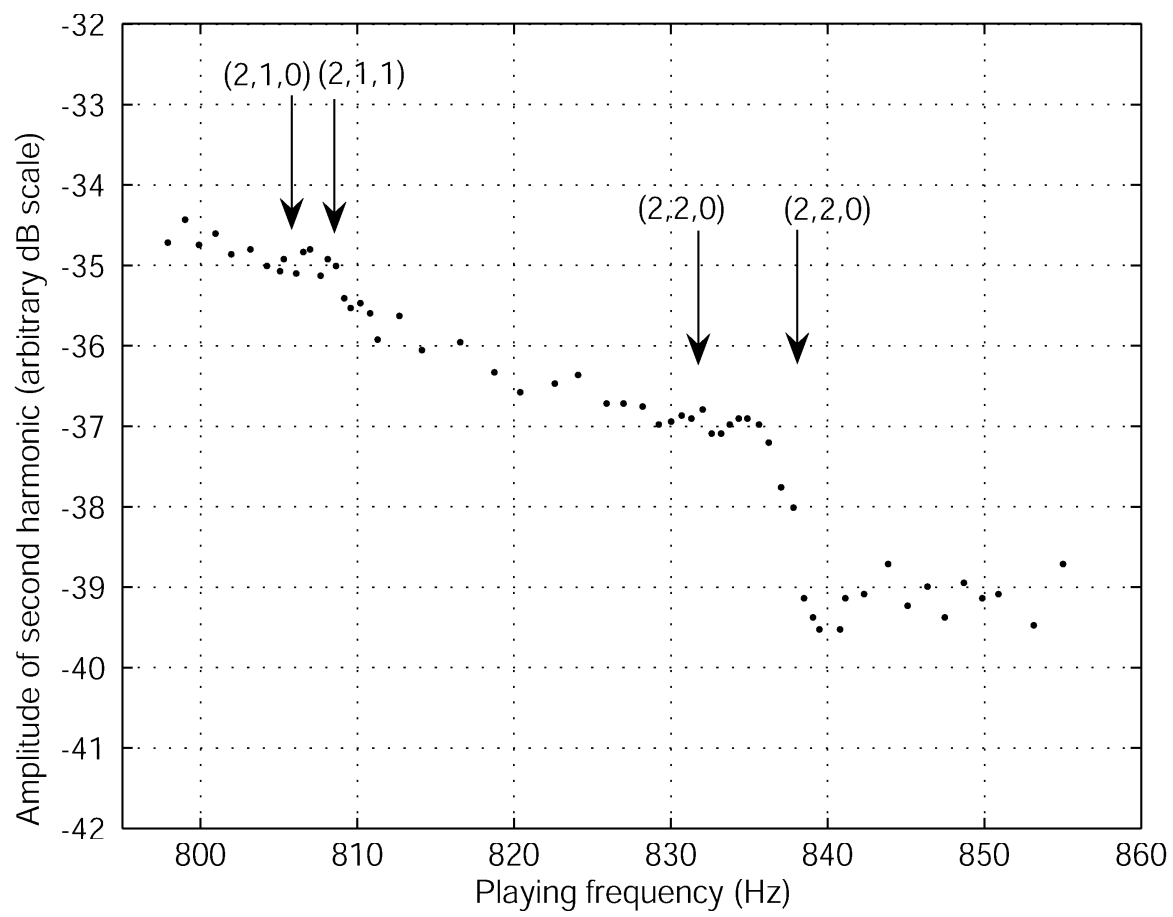

(b)

FIG. 11. 


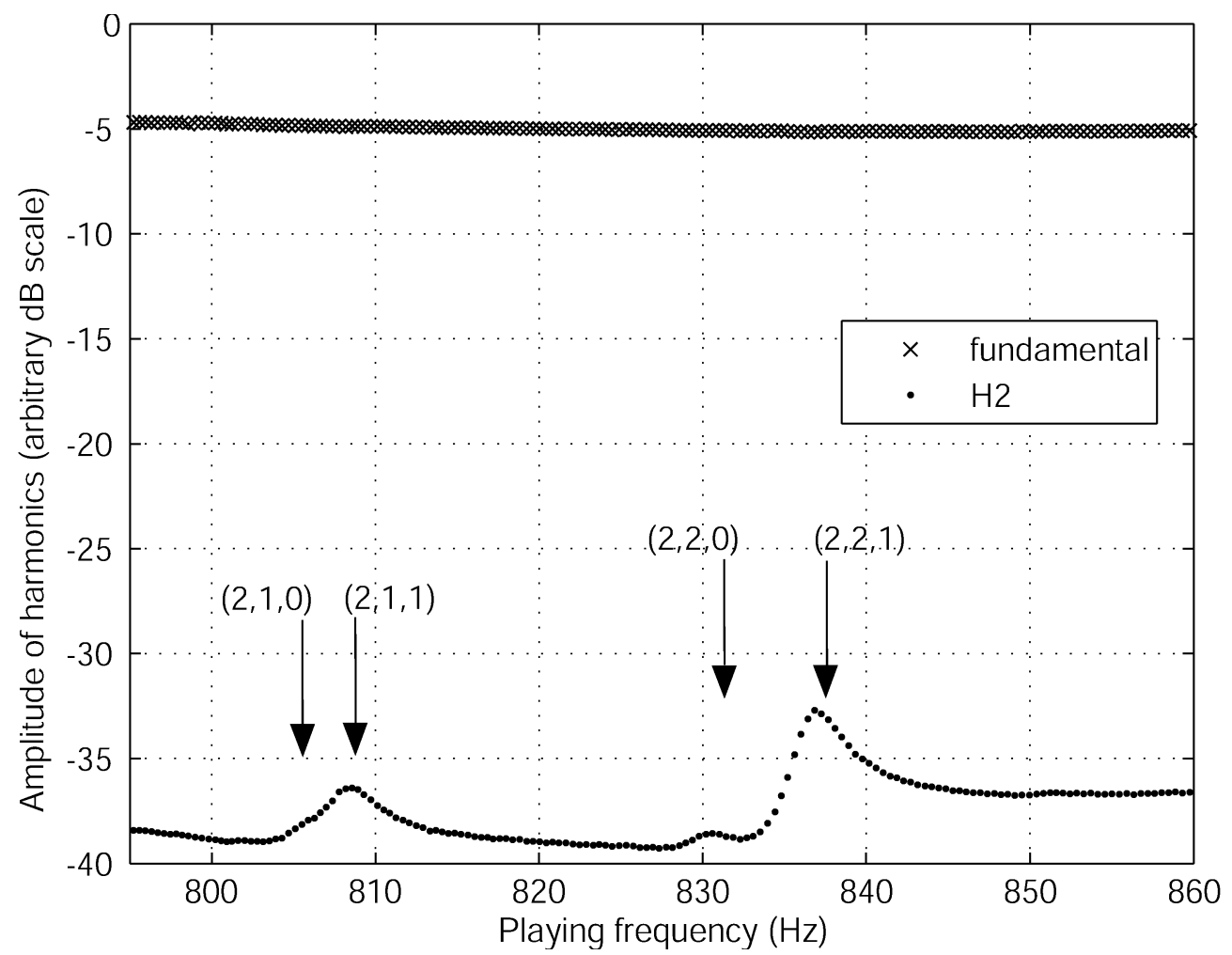

FIG. 12. 